Original paper

(Artículo científico)

\title{
NEW RECORDS OF GENUS CULICOIDES LATREILLE FROM OAXACA, MEXICO (DIPTERA: CERATOPOGONIDAE)
}

\author{
NUEVOS REGISTROS DEL GÉNERO CULICOIDES LATREILLE DE OAXACA, MÉXICO \\ (DIPTERA: CERATOPOGONIDAE)
}

\author{
HERÓN HUERTA ${ }^{1^{*}}$, JOSÉ ISMAEL BENÍTEZ-ALVA², JORGE CONCHA-SUAREZ ${ }^{2}$, \\ CLEMENTE R. IBÁÑEZ-PIÑ ÓN ${ }^{2}$, PABLO MANRIQUE-SAIDE ${ }^{3}$
}

\begin{abstract}
${ }^{1}$ Laboratorio de Entomología, Instituto de Diagnóstico y Referencia Epidemiológicos. Francisco de P. Miranda No. 177, Col. Unidad Lomas Plateros, DT. Álvaro Obregón, CP. 01480, Ciudad de México, México. <cerato_2000@yahoo.com> ${ }^{2}$ Departamento de Control de Enfermedades Transmitidas por Vectores. 20 noviembre \#707, Centro, 68000, Oaxaca de Juárez, México.<biol.benitez.alva@gmail.com>; <vectoresso@gmail.com>; <cleraf@hotmail.com> ${ }^{3}$ Unidad Colaborativa para Bioensayos Entomológicos, Campus Ciencias Biológicas y Agropecuarias, Universidad Autónoma de Yucatán, Mérida, Yucatán, México. <pablo_manrique2000@hotmail.com>

*Corresponding author: <cerato_2000@yahoo.com>
\end{abstract}

Received: 04/03/2020; accepted: 07/10/2020; published online: 14/10/2020

Responsible editor: Karina D. Rivera-García

Huerta, H., Benítez-Alva, J. I., Concha-Suarez, J., Ibáñez-Piñón, C. R., Manrique-Saide, P. (2020) New records of genus Culicoides Latreille from Oaxaca, Mexico (Diptera: Ceratopogonidae). Acta Zoológica Mexicana (nueva serie), 36, 1-27.

https://doi.org/10.21829/azm.2020.3612280

\begin{abstract}
First records of Culicoides ginesi and Culicoides glabellus for Mexico. New distribution records for the state of Oaxaca of the following species are also presented: Culicoides baueri, Culicoides debilipalpis, Culicoides hylas, Culicoides neopulicaris, Culicoides pseudodiabolicus and Culicoides pusillus. Also, new localities for Culicoides foxi, Culicoides insignis, Culicoides jamaicensis and Culicoides ocumarensis are included. A key and table are presented for the known species in the state of Oaxaca.
\end{abstract}

Key words: Culicoides; new record; neotropics; key

Huerta, H., Benítez-Alva, J. I., Concha-Suarez, J., Ibáñez-Piñón, C. R., Manrique-Saide, P. (2020) Nuevos registros del género Culicoides Latreille de Oaxaca, México (Diptera: Ceratopogonidae). Acta Zoológica Mexicana (nueva serie), 36, 1-27. https://doi.org/10.21829/azm.2020.3612280

RESUMEN. Se incluye el primer registro de Culicoides ginesi y Culicoides glabellus para México. Se dan a conocer nuevos registros de distribución para el estado de Oaxaca de las siguientes especies: Culicoides baueri, Culicoides debilipalpis, Culicoides hylas, Culicoides neopulicaris, Culicoides pseudodiabolicus y Culicoides pusillus. Asimismo, se incluyen nuevas localidades para Culicoides foxi, Culicoides insignis, Culicoides jamaicensis y Culicoides ocumarensis. Se presenta una clave y un cuadro para las especies conocidas en el estado de Oaxaca. 
Palabras clave: Culicoides; nuevo registro; neotrópico; clave

\section{INTRODUCTION}

The biting midges of the genus Culicoides Latreille include a diverse group widely distributed worldwide, which occur in varied habitats from coastal areas to high altitudes (Mellor et al., 2000; Spinelli \& Borkent, 2004). Culicoides genus is the most diverse within the Ceratopogonidae family, with almost 1,347 recognized extant species in the world (Borkent \& Dominiak, 2020). Various species have medical and veterinary importance and role as biological vectors of viruses, protozoa, and filarial nematodes (Borkent, 2005; Borkent \& Spinelli, 2007), and others are known as notorious pests of humans and other warmblooded vertebrates (Kettle, 1977; Linley et al.,1983; Borkent, 2005; Borkent \& Spinelli, 2007).

In Mexico, biting midges are commonly known as "jejenes" or "chaquistes". Early descriptions by Dampf (1936), recognized them as a pest, and several specimens were identified with filarial nematodes in the state of Chiapas, south of Mexico. Other studies related them to the transmission of mansonelliasis in the Peninsula of Yucatan (Biagi et al., 1958).

Current knowledge of the genus Culicoides in Mexico includes 84 species (11 endemic) belonging to 14 subgenera, nine species groups and four species without any group or subgenus (Ibáñez-Bernal et al., 1996; Borkent \& Spinelli, 2000; 2007; Huerta et al., 2012; Spinelli \& Huerta, 2015). However, some regions of Mexico are still scantily studied. This is the case of the states of Aguascalientes, Guanajuato and Tlaxcala that do not have any record for Culicoides species (Ibáñez-Bernal et al., 1996).

Here we report recent collections performed at the state of Oaxaca, with two new records for Mexico, six new records from the state of Oaxaca and new local records for another four previously reported species. Additionally, we include an updated list of known species for the state of Oaxaca (Table 1) and a key to separate the species from this state.

\section{MATERIALS AND METHODS}

Adults specimens were collected by CDC light traps, and then preserved in ethanol $70 \%$. Subsequently, specimens were cleared, dissected, and mounted on microscope slides in Canada balsam using the technique described by Borkent and Spinelli (2007). Most of the specimens were mounted on microscope slides in Canada balsam, a set of remaining specimens was preserved directly in ethanol $70 \%$. The specimens were examined and measured with a binocular compound microscope Olympus BX50. The photographs were taken with a Lumenera ${ }^{\circledR}$ Infinity 1, digital camera attached to Olympus BX50 compound microscope and SZX7 stereoscopic. The photos were edited using Adobe Photoshop.

Morphological terminology is based on Borkent (2017) and Borkent et al. (2009). Assignment of species to subgenus and species groups follows the system proposed by Borkent (2016). Regardless of how collection data of specimens is presented on specimen labels, this information is presented here in the following order: state, locality/localities data, date(s) of collection, name(s) of collector(s), method(s) of collection, number of specimens and their sexes. All specimens examined are deposited in the Colección de Artrópodos con Importancia Médica (CAIM), Mexico City, Mexico.

Abbreviation wing. $\mathrm{CuA}=$ anterior branch of cubital veins; $\mathrm{m}_{1}, \mathrm{~m}_{2}$ and $\mathrm{m}_{4}=$ medial cells; $\mathrm{M}_{1}, \mathrm{M}_{2}$ and $\mathrm{M}_{4}=$ branches of medial vein; $\mathrm{R}_{1}, \mathrm{R}_{3}$, = radial veins; $\mathrm{r}-\mathrm{m}=$ radial-medial cross vein; $\mathrm{r}_{1}, \mathrm{r}_{2}$ and $\mathrm{r}_{3}=$ radial cells; according to Borkent (2017). 


\section{RESULTS}

Table 1. List records of Culicoides from Oaxaca, Mexico.

\begin{tabular}{|c|c|c|}
\hline Species & Locality & Record \\
\hline Culicoides baueri+ & Santiago Yaveo & in the present \\
\hline Culicoides copiosus & $\begin{array}{l}\text { Comatlán, Ixtlán } \\
\text { Etla }\end{array}$ & Vargas, 1945 \\
\hline Culicoides diabolicus & San Juan Guichicovi, El Zarzal & Huerta et al., 2012 \\
\hline Culicoides debilipalpis+ & Santiago Yaveo & in the present \\
\hline Culicoides elutus & Camotlán, Ixtlán & Wirth \& Blanton, 1959 \\
\hline Culicoides gabaldoni & San Juan Guichicovi, El Zacatal & Huerta et al., 2012 \\
\hline Culicoides ginesi* & Santiago Yaveo & in the present \\
\hline Culicoides glabellus* & Santiago Yaveo & in the present \\
\hline \multirow{3}{*}{ Culicoides foxi } & $\begin{array}{l}\text { Rio Jaltepec } \\
\text { Istmo de Tehuantepec }\end{array}$ & Spinelli et al., 1993 \\
\hline & San Juan Guichicovi, El Zacatal & Huerta et al., 2012 \\
\hline & $\begin{array}{l}\text { Santiago Yaveo, } \\
\text { Putla Villa de Guerrero, Rancho Viejo }\end{array}$ & in the present \\
\hline Culicoides furens & without locality data & Wirth \& Blanton, 1974 \\
\hline Culicoides hylas+ & Santiago Yaveo & in the present \\
\hline \multirow[t]{2}{*}{ Culicoides insignis } & $\begin{array}{l}\text { Arroyo Chopan, Tuxtepec } 20 \mathrm{Km} \mathrm{S} \text {. } \\
\text { Bethania } \\
\text { Rio Jaltepec, } \\
\text { Istmo de Tehuantepec }\end{array}$ & Spinelli et al., 1993 \\
\hline & Santiago Yaveo & in the present \\
\hline \multirow{2}{*}{ Culicoides jamaicensis } & San Juan Guichicovi, El Zarzal & Huerta et al., 2012 \\
\hline & Santa Cruz Zenzontepec, Soledad Cofradía & in the present \\
\hline Culicoides leopoldoi & San Juan Guichicovi, El Zarzal & Huerta et al., 2012 \\
\hline Culicoides luteovenus & Yolox, Ixtlán & Vargas, 1945 \\
\hline Culicoides neopulicaris+ & $\begin{array}{l}\text { Santa María Tonameca, } \\
\text { Arroyo La Puerta }\end{array}$ & in the present \\
\hline \multirow[t]{2}{*}{ Culicoides ocumarensis } & $\begin{array}{l}\text { Rio Jaltepec, } \\
\text { Istmo de Tehuantepec }\end{array}$ & Spinelli et al., 1993 \\
\hline & Santiago Yaveo & in the present \\
\hline Culicoides pampoikilus & without locality data & Borkent \& Spinelli, 2000 \\
\hline Culicoides phlebotomus & Salina Cruz & Wirth \& Blanton, 1953 \\
\hline Culicoides pusillus+ & $\begin{array}{l}\text { Santa Cruz Zenzontepec, Soledad Cofradía } \\
\text { Santiago Yaveo }\end{array}$ & in the present \\
\hline $\begin{array}{l}\text { Culicoides } \\
\text { pseudodiabolicus+ }\end{array}$ & Santiago Yaveo & in the present \\
\hline Culicoides rangeli & Comatlán, Ixtlán & $\begin{array}{l}\text { Vargas, } 1954 \text { (as } \\
\text { donajii) } \\
\text { Wirth, } 1974\end{array}$ \\
\hline Culicoides stigmalis & Río Cajones, entre Yaé y Comatlán & $\begin{array}{l}\text { Vargas, } 1953 \\
\text { Wirth \& Blanton, } 1959\end{array}$ \\
\hline Culicoides variipennis & Etla & Vargas, 1945 \\
\hline
\end{tabular}




\section{Key to the Culicoides species from Oaxaca, Mexico (Primarily for females)}

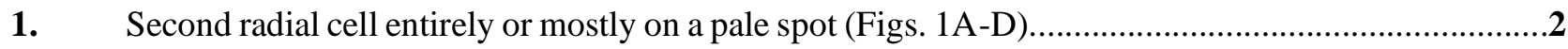

- Second radial cell entirely on a dark spot (Figs. 3A-D) ............................................................10

2(1) Base of cell $\mathrm{m}_{4}$ pale bordering veins $\mathrm{M}_{4}$ and $\mathrm{CuA}$, or apices of veins $\mathrm{M}_{1}$ and $\mathrm{M}_{2}$ pale.....................5

- $\quad$ Base of cell $\mathrm{m}_{4}$ and adjacent veins on a dark area; apices of veins $\mathrm{M}_{1}$ and $\mathrm{M}_{2}$ dark...........................3

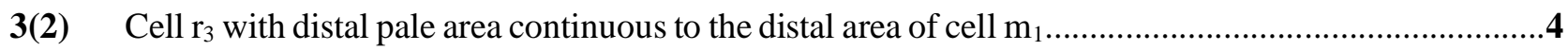

- Cell $\mathrm{r}_{3}$ with distal pale area discontinuous to the distal area of cell

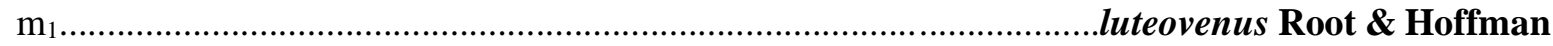

4(3) Cell $\mathrm{m}_{4}$ with a small dark area in the middle (Fig. 2C)........................................neopulicaris Wirth

- $\quad$ Cell $\mathrm{m}_{4}$ without a small dark area in the middle................................................................elutus Macfie

5(2) Cell $r_{3}$ with a separate pale spot beyond to the base of vein $M_{1}$ (hylas species group) (Fig. 1B); third palpal segment with a subdivided sensory pit................................................................hylas Macfie Cell $r_{3}$ without a separate pale spot beyond to the base of vein $M_{1}$, pale area continuous from $r-m$ cross-vein to the borders of vein $\mathrm{M}_{1}$ (guttatus species group) (Fig. 1A); third palpal segment with or without a subdivided sensory pit.

6(5) Cross-vein $r-m$ pale. .7

- $\quad$ Cross-vein r-m dark .8

7(6) Halter knob dark; vein $\mathrm{M}_{2}$ with discontinuous straddling pale area (Fig. 2A); nineth tergite of the male with mesal cleft, and the apicolateral process is very close ocumarensis Ortiz

- $\quad$ Halter knob pale; vein $\mathrm{M}_{2}$ with continuous straddling pale area; nineth tergite of the male without mesal cleft, and the apicolateral process is widely spaced. diabolicus Hoffman

8(6) Cell $\mathrm{m}_{1}$ with a distal pale spot; halter dark; vein $\mathrm{R}_{3}$ dark up to the point where it turns abruptly forward to meet with costal vein (Fig. 1D)..... insignis Lutz

- $\quad$ Cell $\mathrm{m}_{1}$ with two distal pale spot, halter dark or pale; vein $\mathrm{R}_{3}$ pale. ...9

9(8) Halter knob pale; third palpal segment with subdivided pit; vein $\mathrm{R}_{3}$ pale (infuscated for a short distance in some specimens) (Fig. 1C) .pseudodiabolicus Fox Halter knob dark brown; third palpal segment with shallow and rounded pit; vein $\mathrm{R}_{3}$ pale but with small blackish spot behind apex (Fig. 1A). foxi Ortiz

10(1) Wing with contrasting pattern of dark and pale spot (Fig. 3) .12

Wing with very faint pattern (Fig. 2B)

11(10) Cell $r_{2}$ with similar proportion to the $r_{1}$ (Fig. 2B); hind tibial comb with five spines; halter yellowish. .pusillus Lutz Cell $\mathrm{r}_{2}$ longer to the cell $\mathrm{r}_{1}$; hind tibial comb with four spines; halter dark. stigmalis Wirth

12(10) Eyes widely separated; spermatheca $U$ or C-shaped (subgenera Monoculicoides). variipennis (Coquillett)

Eyes slightly separated; spermatheca different shaped 
13(12) Wing with a pale straddling middle of vein $M_{2}$ (Fig. 2D), or veins $M_{1}$ and $M_{2}$ with completely pale margins.

- Wing without a pale straddling middle of vein $\mathrm{M}_{2}$, or veins $\mathrm{M}_{1}$ and $\mathrm{M}_{2}$ without completely pale margins, usually dark to apex.

14(13) Third palpal segment with a very large and deep sensory pit

15(14) Base of vein $M_{1}$ with straddling pale spot.

16(15) Apex of the veins $M_{1}$ and $M_{2}$ with a pale area in the wing margin (Fig. 2D); cell $r_{3}$ with distal pale area reaching the wing margin jamaicensis Edwards

- $\quad$ Apex of the veins $M_{1}$ and $M_{2}$ without a pale area in the wing margin; cell $r_{3}$ with distal pale area not reaching the wing margin. copiosus Root \& Hoffman

17(14) Mesonotum with pattern of punctiform brown dots; cell $\mathrm{m}_{1}$ with three pale spots, the distal at the wing margin furens (Poey) Mesonotum without pattern of punctiform brown dots; cell $\mathrm{m}_{1}$ with one pale spots, located far from wing margin

18(17) Legs with distinct pale bands; hind tibial comb with four spines; wing with abundant macrotrichia (Fig. 3A). baueri Hoffman Legs without distinct pale bands; hind tibial comb six to eight spines; wing without abundant microtrichia. phlebotomus (Williston)

19(13) Mesonotum with distinctive pattern of punctiform brown dots; cell $r_{3}$ with three small rounded equidistant pale spots in a triangle (Fig. 3C) Mesonotum without distinctive pattern of punctiform brown dots; cell $r_{3}$ with different arrangement.

20(19) Macrotrichia sparce, only a few in apices of cells $r_{3}, m_{1}$ and $m_{2}$; small species (wing length $0.80 \leq$ $\mathrm{mm})$

Macrotrichia more numerous, present at least on distal half of wing; large species (wing length $0.8 \geq$ $\mathrm{mm})$.

21(20) Two spermathecae; legs paler; cell $\mathrm{r}_{2}$ closed.

gabaldoni Ortiz

- $\quad$ One spermatheca; legs darker; cell $\mathrm{r}_{2}$ open (Fig. 3D) glabellus Wirth \& Blanton

22(20) Two spermathecae; mesonotum with median anterior triangular marking One spermatheca; mesonotum with different pattern debilipalpis Lutz

Cell $r_{3}$ with fourpale spots...

23(22) Cell $r_{3}$ with four pale spots.

Cell $r_{3}$ with two pale spots.......

leopoldoi Ortiz rangeli Ortiz \& Mirsa 


\section{New records for Mexico}

\section{Culicoides (Haematomyidium) ginesi Ortiz}

(Figs. 3C, 4)

Culicoides ginesi Ortiz, 1951b: 586 (Venezuela; female; figs.). Wirth \& Blanton, 1959: 450 (Panama; redescription; debilipalpis group distribution; in key; figs.); Aitken et al., 1975: 126 (Trinidad record; distribution; in key; fig.); Borkent \& Wirth, 1997: 69 (World species of Ceratopogonidae).

Culicoides (Oecacta) ginesi Ortiz: Forattini, 1957: 395 (Brazil; redescription; in key; distribution; figs.); Wirth, 1974: 31 (Catalogue of the Americas south of US).

Culicoides (Haematomyidium) ginesi Ortiz: Wirth et al., 1988: 48 (Wing Atlas of Neotropical species; fig.); Borkent \& Spinelli, 2000: 32 (Catalog of New World, South USA); Spinelli et al., 2005: 141 (Argentina record; in key; fig.); Borkent \& Spinelli, 2007: 66 (Neotropical catalog); Borkent, 2016: 16 (online the subgeneric classification of species of Culicoides); Spinelli \& Wolff, 2016: 110 (Colombia record); Santarém \& Felippe-Bauer, 2020: 23 (Brazilian species); Borkent \& Dominiak, 2020: 113 (World catalogue).

Remarks. This species is widely distributed in the Neotropics and was originally described from Venezuela (Ortiz, 1951b). In Mexico, other similar species belonging to this subgenus, and known are: Culicoides debilipalpis Lutz, Culicoides eadsi Wirth \& Blanton and Culicoides paraensis (Goeldi). Culicoides ginesi can be distinguished from the previous species by the characteristic pattern of strikingly marked of mesonotum and wing with cell $\mathrm{R}_{3}$ well-separated spots arranged in a triangle (Fig. 3C). We examined two females from Oaxaca, which represent the first record from Mexico.

Specimens examined. The examination of a specimen identified by Willis Wirth, deposited in the collection of Ceratopogonidae (CCER/FIOCRUZ) was measurement and photograph by comparison through the courtesy of Dr. Maria Luiza Felippe-Bauer.

New record for Mexico. Oaxaca, Santiago Yaveo (Fig. 4), GPS: $17^{\circ} 20^{\prime}$ 8.5” N, 95 41’ 38” W; 20 Nov. 2019, col. Benitez-Alva, J. I., Reyes-Garcia, F., trap CDC, 2 females (slide mounted).

Distribution. Mexico (Oaxaca). El Salvador to Panama, Colombia, Venezuela, Trinidad, Brazil northeastern Argentina (Borkent \& Spinelli, 2007).

Subgenus unplaced, leoni species group

\section{Culicoides glabellus Wirth \& Blanton \\ (Figs. 3D, 4)}

Culicoides glabellus Wirth \& Blanton, 1956c: 47 (Panama; Honduras; Nicaragua; Trinidad; male; female; distribution; figs.). Wirth \& Blanton, 1959: 429 (Panama; redescription; in key; distribution; figs.); Williams, 1964: 462 (Trinidad; larvae habitat); Wirth et al., 1988: 52 (Wing Atlas of Neotropical species; fig.); Borkent \& Wirth, 1997: 69 (World species of Ceratopogonidae); Borkent \& Spinelli, 2000: 39 (Catalog of New World, South USA); Spinelli \& Borkent, 2004: 393 (Costa Rica records); Borkent \& Spinelli, 2007: 73 (Neotropical catalog); Borkent, 2016: 34 (online the subgeneric classification of species of Culicoides); Spinelli \& Wolff, 2016: 117 (Colombia records); Santarém \& Felippe-Bauer, 2020: 28 (Brazilian species); Borkent \& Dominiak, 2020: 132 (World catalogue).

Culicoides (Oecacta) glabellus Wirth \& Blanton: Wirth \& Blanton, 1973: 437 (Brazil; diagnosis; distribution; in key; fig.); Wirth, 1974: 31 (Catalogue of the Americas south of US); Aitken et al., 1975: 126 (Trinidad record; distribution; in key; fig.). 

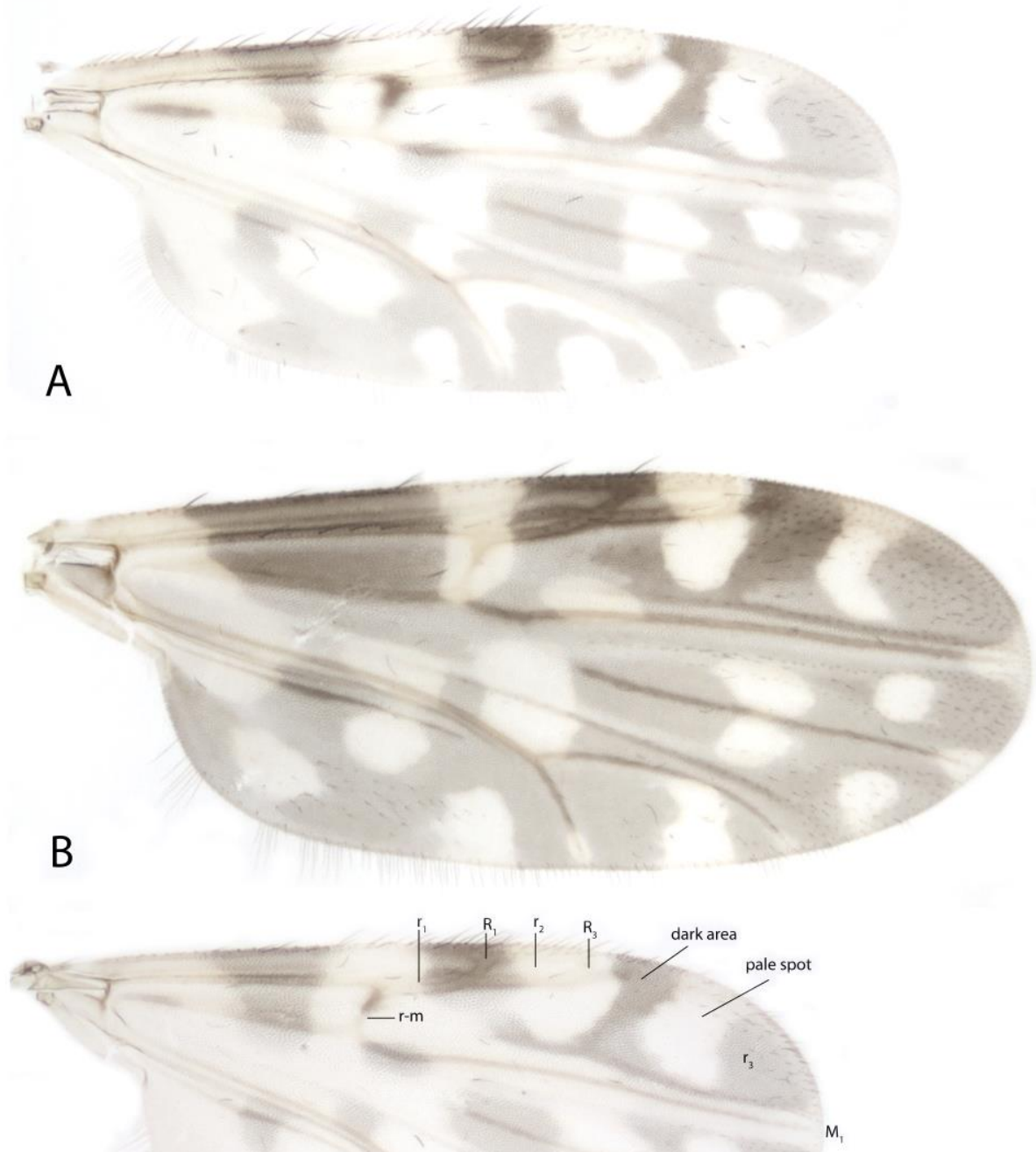

m
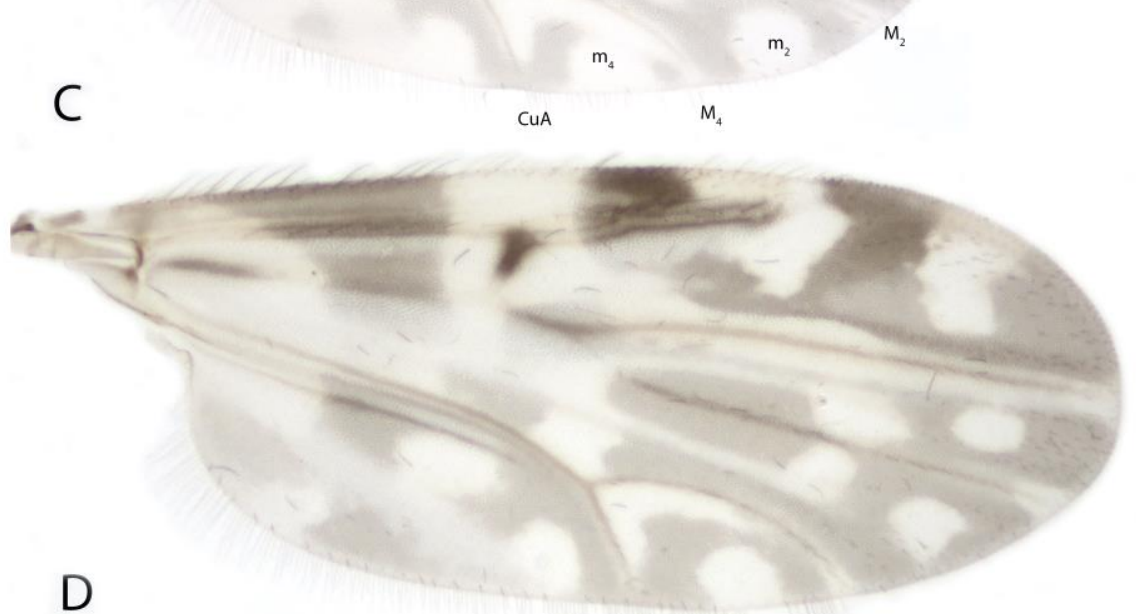

Figure 1. Wing, dorsal view. Female. A) Culicoides foxi Ortiz; B) Culicoides hylas Macfie; C) Culicoides pseudodiabolicus Fox; D) Culicoides insignis Lutz. Scale $=0.5 \mathrm{~mm}$. 
Remarks. According to Borkent (2016), leoni species group included six species from the Neotropical region (Culicoides benarrochi Ortiz \& Mirsa, Culicoides fieldi Wirth \& Blanton, Culicoides gabaldoni Ortiz, Culicoides glabellus Wirth \& Blanton, Culicoides leoni Barbosa and Culicoides trifidus Spinelli \& Borkent and only Culicoides reevesi had distribution in the Nearctic region. The previously known members of the leoni species group from Mexico was C. gabaldoni reported by Wirth \& Blanton (1973), this species is mostly and widely distributed in the Neotropical region (Borkent \& Spinelli, 2007). Huerta et al., (2012) included the first record of $C$. leoni from Mexico (Veracruz). From the samples collected from Oaxaca, we examined two females conspecific to $C$. glabellus, based on the description and key of Wirth \& Blanton (1956c; 1959), which represent the first record from Mexico (Oaxaca).

General appearance, size, and wing markings of $C$. glabellus are very similar to $C$. leoni and $C$. gabaldoni (Wirth \& Blanton, 1959). This last species has two spermathecae, while C. leoni and C. glabellus only present one spermatheca. In C. leoni the distal sensilla coeloconicae present on segments I and V-VIII, and the antennal ratio is 0.75 , compared with $C$. glabellus has the distal sensilla coeloconicae present on segments I and VI-VIII, and antennal ratio is $0.91(0.81-0.95)$.

Specimens examined. PANAMA: Canal Zone, Mojinga Swamp, light trap, 14 Nov. 1951, F. S. Blanton, 1 female (slide) (CAIM Crt/lam-00669).

New record for Mexico. Oaxaca, Santiago Yaveo (Fig. 4), GPS: 17²0' 8.5” N, 95 41' 38” W; 20 Nov. 2019, col. Benitez-Alba, J. I., Reyes-Garcia, F., trap CDC, 2 males (slide mounted); same data except, 23 Oct. 2019, 6 females, 4 males (slide mounted).

Distribution. Mexico (Oaxaca). Honduras to Colombia, Ecuador, Trinidad and Tobago, Brazil (Borkent \& Spinelli, 2007).

\section{New distributional records of species previously recorded from Mexico.}

\section{Culicoides (Avaritia) pusillus Lutz}

(Figs. 2B, 4)

Culicoides pusillus Lutz, 1913: 52 (Brazil; male; female; fig.). Macfie, 1938: 165 (Trinidad record; fig.); Adamson, 1939: 81 (Trinidad; habitats); Adamson, 1941: 75 (Trinidad; habitats); Barbosa, 1947: 25 (Panama; Jamaica records; in key; fig.); Macfie, 1948: 79 (Mexico record; in key); Ortiz \& Mirsa, 1951: 603 (Venezuela record; figs.); Ortiz \& León, 1955: 570 (Ecuador record); Wirth, 1955b: 110 (Guatemala record; fig.); Beck, 1956: 134 (USA, Florida record); Forattini, 1957: 284 (Brazil; redescription; in key; distribution; figs.); Beck, 1958: 11 (USA, Florida record); Williams, 1964: 462 (Trinidad; larvae habitat); Smith \& Varnell, 1967: 519 (USA, Florida; tree holes); Gutsevich et al., 1969: 2 (Cuba; notes); Tikasingh, 1972: 447 (Trinidad; habits); Winder \& Silva, 1972: 653 (Brazil; larval habits); Winder, 1977: 60 (Brazil; larval habits); Mellor et al., 2000: 313 (epidemiology); Borkent \& Wirth, 1997: 80 (World species of Ceratopogonidae); Ronderos \& Spinelli, 1998: 82 (Paraguay record; in key); Silva et al., 2001: 353 (Brazil record); Soria et al., 2002: 320 (Brazil record).

Culicoides (Avaritia) pusillus Lutz: Wirth \& Blanton, 1959: 292 (Panama; redescription; distribution; in key; figs.); Wirth \& Blanton, 1973: 446 (Brazil; diagnosis; in key; distribution; fig.); Wirth, 1974: 21 (Catalogue of the Americas south of US); Wirth \& Blanton, 1974: 77 (West Indian review; distribution; in key; figs.); Aitken et al., 1975: 138 (Trinidad record; distribution; in key; fig.); Blanton \& Wirth, 1979: 140 (USA, Florida; diagnosis; distribution; in key; figs); Wirth et al., 1988: 14 (Wing Atlas of Neotropical species; fig.); Wirth \& Mullens, 1992: 1007 (in key; pusillus group); Borkent \& Spinelli, 2000: 28 (Catalog of New World, South USA); Ronderos et al., 2003: 22 (Paraguay record); Spinelli \& Borkent, 2004: 390 (Costa Rica records); Spinelli et al., 2005: 138 
(Argentina record; in key; fig.); Borkent \& Spinelli, 2007: 63 (Neotropical catalog); Spinelli et al., 2009: 87 (Colombia record); Huerta et al., 2012 (Mexico records); Borkent, 2016: 9 (online the subgeneric classification of species of Culicoides); Spinelli \& Wolff, 2016: 108 (Colombia record); Santarém \& Felippe-Bauer, 2020: 22 (Brazilian species); Borkent \& Dominiak, 2020: 105 (World catalogue).

Remarks. This species is widely distributed from United States (Florida) and Mexico, south to Brazil and northeastern of Argentina (Borkent \& Spinelli, 2000; 2007). In Mexico it was previously reported from states of Chiapas, Tabasco, and Veracruz (Macfie, 1948; Huerta et al., 2012).

New record for Oaxaca. Oaxaca, Santiago Yaveo (Fig. 4), GPS: $17^{\circ} 20^{\prime} 8.5^{\prime \prime}$ N, 95 41' 38” W; 23 Nov. 2019, col. Benitez-Alva, J. I., Reyes-Garcia, F., trap CDC, 1 male (slide mounted); Santa Cruz Zenzontepec, La Soledad Cofradía, GPS: $16^{\circ} 32$ ' 40.4” N, 97 32' 44.9” W; 26 Nov. 2019, col. Benitez-Alva, J. I., PerezMartinez, D., trap CDC, 1 male (slide mounted).

Distribution. United States, Mexico (Chiapas, Tabasco, Veracruz, Oaxaca) to northeastern of Argentina (Borkent \& Spinelli, 2007; Huerta et al., 2012).

\section{Culicoides (Culicoides) neopulicaris Wirth}

(Figs. 2C, 4)

Culicoides neopulicaris Wirth, 1955a: 355 (United States; Mexico; female; figs.). Borkent \& Wirth, 1997: 75 (World species of Ceratopogonidae); Borkent, 2014: 208 (pupae reference).

Culicoides (Culicoides) neopulicaris Wirth: Vargas, 1960: 39 (subgenera of Culicoides of America); Wirth, 1965: 128 (Nearctic catalog); Wirth \& Blanton, 1969: 229 (North American review pulicaris group; diagnosis; distribution; in key; figs.); Wirth, 1974: 21 (Catalogue of the Americas south of US); Wirth et al., 1985: 12 (Wing Atlas of Nearctic species; fig.); Borkent \& Spinelli, 2007: 64 (Neotropical catalog); Borkent \& Grogan, 2009: 13 (Nearctic catalog); Vigil et al., 2014: 6 (United States record; distribution); Borkent, 2016: 12 (online the subgeneric classification of species of Culicoides); Ronderos et al., 2018: 639 (immature list); Borkent \& Dominiak, 2020: 109 (World catalogue).

Culicoides yukonensis Hoffman: misidentification, Vargas, 1945: 45 (Mexico record).

Remarks. This species has a widely distribution from United States (Texas, Louisiana, Alabama) to Costa Rica. In Mexico, five species belonging to subgenera Culicoides Latreille are recognized: Culicoides elutus Macfie, Culicoides fortinensis Spinelli \& Huerta, Culicoides luteovenus Root \& Hoffman, Culicoides neopulicaris and Culicoides rulfoi Spinelli \& Huerta. This species has been previously reported from seven states of Mexico (Vargas, 1945; Wirth, 1965; Wirth \& Blanton, 1969; Huerta et al., 2012).

New record for Oaxaca. Oaxaca, Santa María Tonameca, Arroyo La Puerta (Fig. 4), GPS: $15^{\circ} 45^{\prime}$ 51.4" N, 96 42' 44.67" W; 24 Nov. 2019, col. Benitez-Alva, J. I., Zurita-Perez, G., trap CDC, 5 females (2 slide mounted, 3 preserved in ethyl alcohol), 1 male (slide mounted).

Distribution. United States, Mexico (Chiapas, Hidalgo, Estado de Mexico, Morelos, San Luis Potosi, Oaxaca, Veracruz, and Yucatan) to Costa Rica (Borkent \& Spinelli, 2007; Vargas, 1945; Wirth, 1965; Wirth \& Blanton, 1969; Huerta et al., 2012). 
Huerta et al.: Records of Culicoides in Oaxaca, Mexico
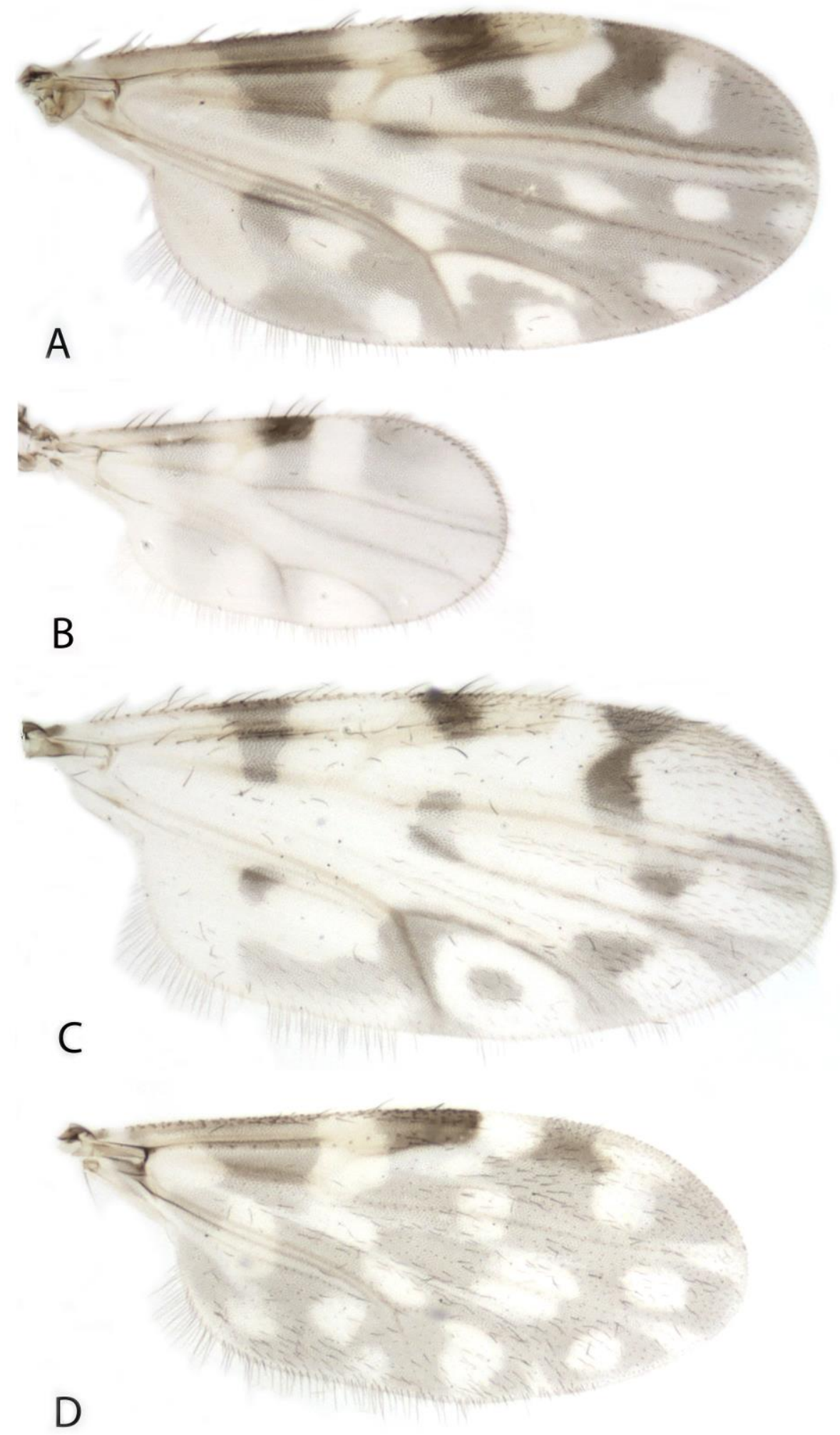

D

Figure 2. Wing, dorsal view. Female. A) Culicoides ocumarensis Ortiz; B) Culicoides pusillus Lutz; C) Culicoides neopulicaris Wirth; D) Culicoides jamaicensis Edwards. Scale $=0.5 \mathrm{~mm}$. 

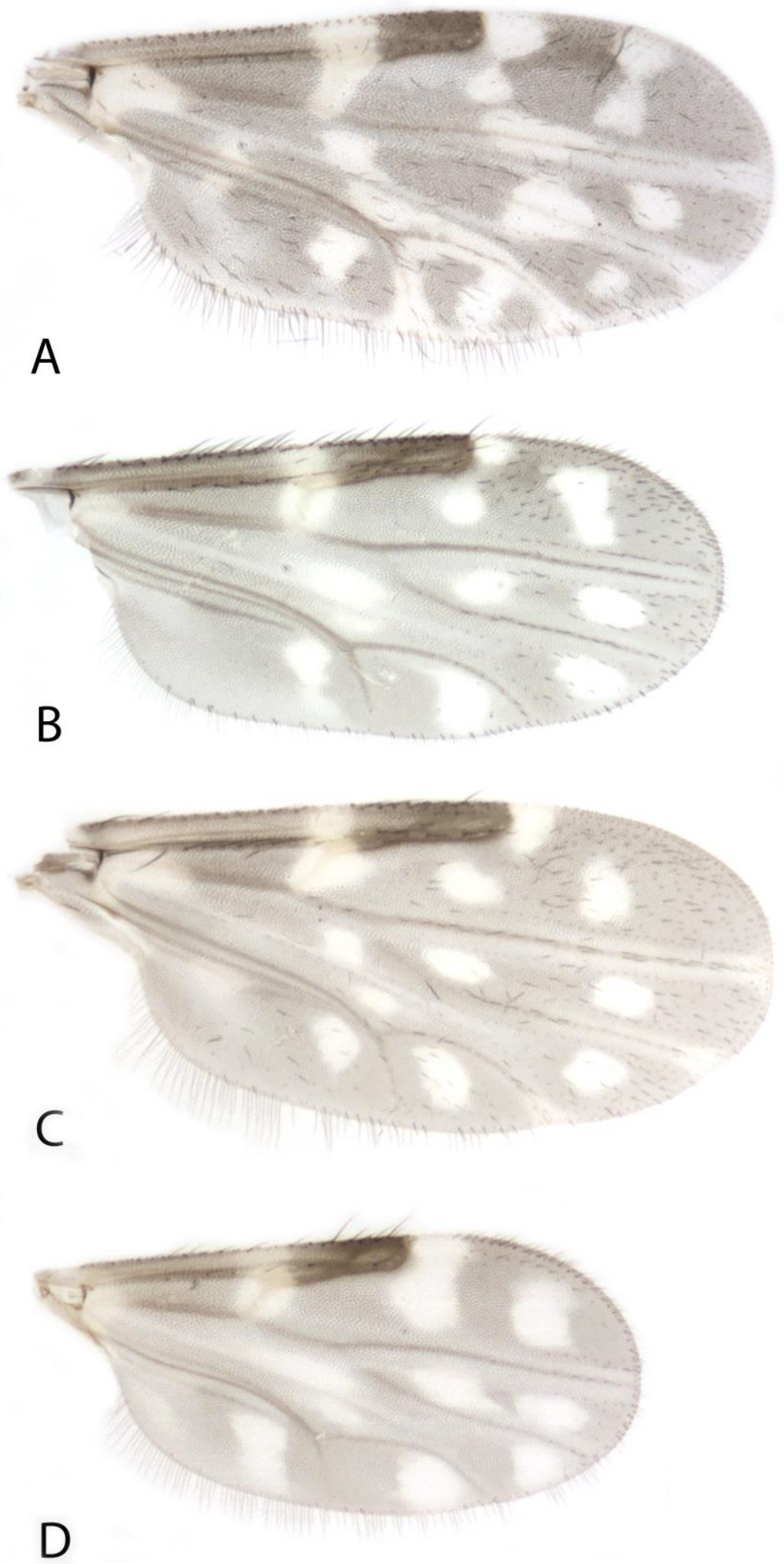

Figure 3. Wing, dorsal view. Female. A) Culicoides baueri Hoffman; B) Culicoides debilipalpis Lutz; C) Culicoides ginesi Ortiz; D) Culicoides glabellus Wirth \& Blanton. Scale $=0.5 \mathrm{~mm}$. 


\section{Culicoides (Diphaomyia) baueri Hoffman}

(Fig. 3A, 4)

Culicoides baueri Hoffman, 1925: 297 (United States; female; figs.). Root \& Hoffman, 1937: 163 (notes; distribution; figs.); Vargas, 1945: 43 (Mexico record); Macfie, 1948: 72 (in key); Vargas, 1949: 195 (list species); Beck, 1952: 104 (USA, Florida record); Foote \& Pratt, 1954: 16 (United States record; diagnosis; distribution; in key; figs.); Williams, 1955: 33 (USA, Georgia; distribution; larval habitat); Messersmith, 1966: 93 (seasonal incidence); Khalaf, 1969: 1159 (distribution; seasonal incidence); Battle \& Turner, 1970: 426 (diagnosis; distribution; in key; larval habitat); Gazeau \& Messersmith, 1970: 32 (habitat); Cochrane, 1974: 315 (distribution; notes); Root \& Gerhardt 1991: 127 (seasonal emergence); Reeves et al., 2004: 8 (USA record; notes); Borkent \& Wirth 1997: 63 (World species of Ceratopogonidae).

Culicoides (Diphaomyia) baueri Hoffman: Wirth, 1965: 131 (Nearctic catalog); Atchley, 1967: 985 (New Mexico; diagnosis; distribution; in key; figs.); Battle \& Turner, 1971: 24 (redescription; distribution; in key; figs.); Blanton \& Wirth, 1979: 61 (as Diphaeomyia (sic); redescription; distribution; in key; figs.); Wirth et al., 1985: 39 (Wing Atlas of Nearctic species; fig.); Borkent \& Grogan, 2009: 34 (Nearctic catalog); Huerta et al., 2012: 2 (Mexico record); Harrup et al., 2015: 251 (fig.); Mukhopadhyay et al., 2016: 281 (India; species list; distribution); Borkent, 2016: 13 (online the subgeneric classification of species of Culicoides); Borkent \& Dominiak, 2020: 110 (World catalogue).

Culicoides (Oecacta) baueri Hoffman: Jamnback, 1965: 42 (United States; figs.).

Remarks. This primarily Nearctic species is distributed from south of the United States (Borkent \& Grogan, 2009). In Mexico, it was previously known from specimens collected by light trap from Mexico City (Root \& Hoffman, 1937). Huerta et al. (2012) confirmed such records from Mexico.

New record for Oaxaca. Oaxaca, Santiago Yaveo (Fig. 4), GPS: $17^{\circ} 20^{\prime}$ 8.5” N, 95 41' 38” W; 22 Oct. 2019, col. Benitez-Alva, J. I., Reyes-Garcia, F., trap CDC, 2 females (slide mounted).

Distribution. Southeastern United States, Mexico (Ciudad de México, Oaxaca, Puebla) (Root \& Hoffman, 1937; Borkent \& Grogan, 2009; Huerta et al., 2012). Mukhopadhyay et al., (2016) indicated this species was recorded from India.

\section{Culicoides (Drymodesmyia) jamaicensis}

(Figs. 2D, 4)

Culicoides loughnani var. jamaicensis Edwards, 1922: 165 (Jamaica; female; fig.). Hoffman, 1925: 283 (Panama record); Vargas, 1945: 43 (Mexico record); Barbosa, 1947: 21 (Panama, Jamaica records; fig.); Macfie, 1948: 80 (Chiapas, Mexico; female; descriptive notes; in key); Vargas, 1949: 200 (list species); Fox, 1949: 32 (Puerto Rico; figs.); Ortiz \& Mirsa, 1952: 271 (in part, Venezuela; figs.); Forattini, 1957: 412 (in part, Brazil; diagnosis; distribution; in key; fig.).

Culicoides jamaicensis Edwards: Wirth, 1955b: 112 (Guatemala record; fig.); Wirth \& Blanton, 1959: 339 (Panama; copiosus group; redescription; distribution; in key; figs.); Wirth \& Hubert, 1960: 649 (review copiosus group; diagnosis; in key; figs.); Williams, 1964: 463 (Trinidad; larvae habitat); Borkent \& Wirth, 1997: 71 (World species of Ceratopogonidae).

Culicoides (Drymodesmyia) jamaicensis Edwards: Wirth, 1974: 22 (Catalogue of the Americas south of US); Wirth \& Blanton, 1974: 61 (West Indian review; diagnosis; distribution; in key; figs.); Aitken et al., 1975: 131 (Trinidad record; distribution; in key; fig.); Wirth et al., 1988: 26 (Wing Atlas of Neotropical species; fig.); Borkent \& Spinelli, 2000: 30 (Catalog of New World, South USA); Spinelli \& Borkent, 2004: 390 (Costa Rica records); Borkent \& Spinelli 2007: 65 (Neotropical catalog); Spinelli et al., 2009: 87 (Colombia records); Huerta et al., 2012: 9 (Mexico records); Borkent, 2016: 14 (online the subgeneric classification of species of Culicoides); Spinelli \& Wolff, 2016: 109 
(Colombia records); Santarém \& Felippe-Bauer, 2020: 22 (Brazilian species); Borkent \& Dominiak, 2020: 111 (World catalogue).

Remarks. This species has a wide Neotropical distribution, including part of the Nearctic region of United States (Borkent \& Grogan, 2009). Macfie (1948) included the first record of C. jamaicensis in Mexico from Chiapas. Huerta et al. (2012) provided records from the states of Guerrero, Jalisco, Estado de Mexico, Oaxaca, Veracruz, and Yucatán.

New record for Oaxaca. Santa Cruz Zenzontepec, La Soledad Cofradía (Fig. 4), GPS: $16^{\circ} 32^{\prime} 40.4^{\prime \prime}$ N, 97 32' 44.9”' W; 26 Nov. 2019, col. Benitez-Alva, J.I., Perez-Martinez, D., trap CDC, 4 females, 3 males (slide mounted). (Fig. 4).

Distribution. United States (Texas, Florida), Mexico (Jalisco, Estado de Mexico, Veracruz, Guerrero, Chiapas, Oaxaca, and Yucatan), Central America and Caribbean to Colombia, Venezuela, and Brazil (Borkent \& Spinelli, 2007; Huerta et al., 2012).

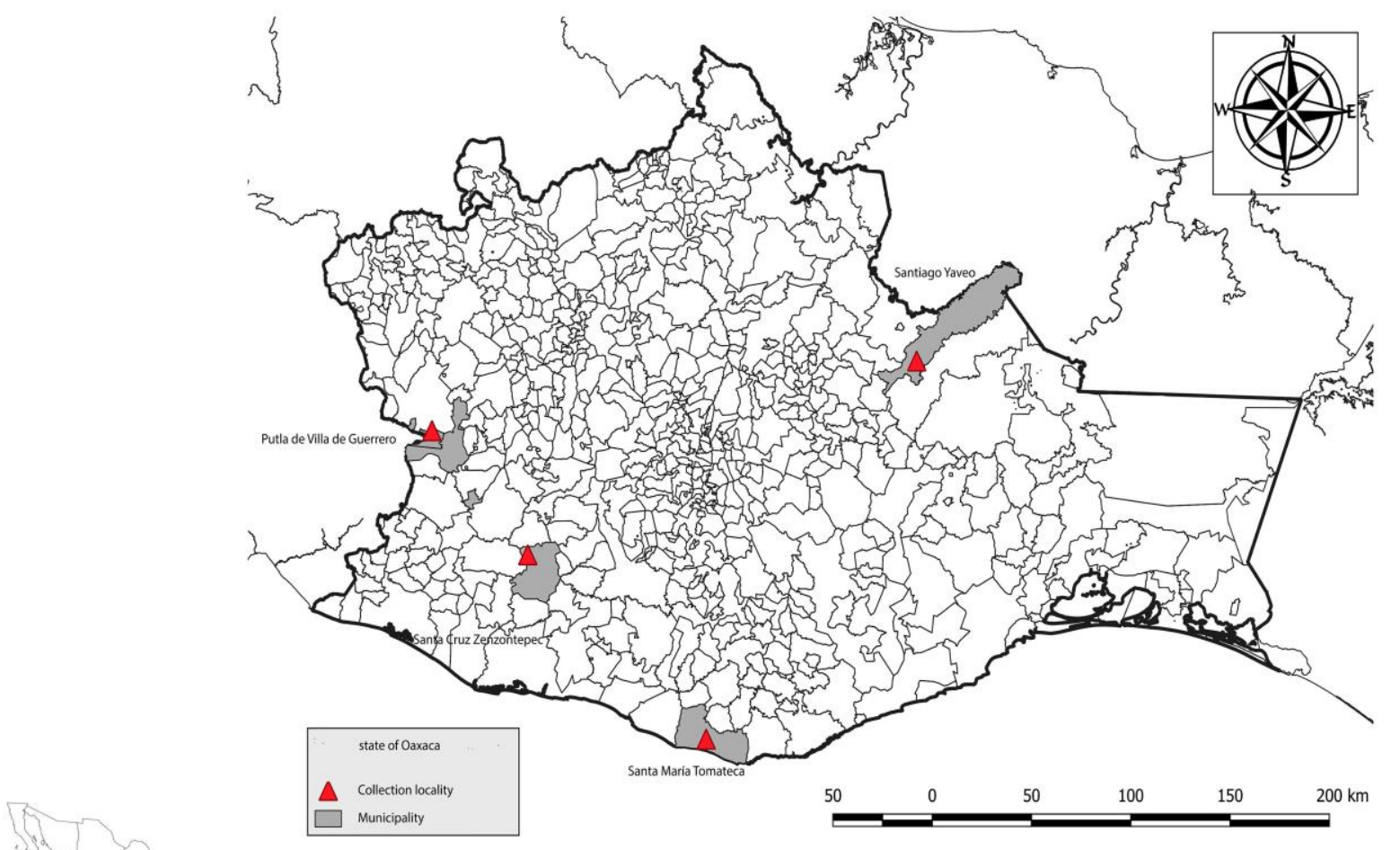

Figure 4. Map of Mexico, with the collection records in the state of Oaxaca. 


\section{Culicoides (Haematomyidium) debilipalpis Lutz \\ (Figs. 3B, 4)}

Culicoides debilipalpis Lutz, 1913: 60 (Brazil; female; description; fig.); Macfie, 1937: 7 (Trinidad record; female; redescription); Floch \& Abonnenc, 1942: 3 (French Guiana record); Vargas, 1945: 43 (Mexico record); Macfie, 1948: 78 (Caribbean record; in key); Vargas, 1949: 197 (list species); Barbosa, 1952: 12 (Argentina, Brazil records); Forattini, 1957: 383 (in part, Brazil; redescription; distribution; in key; figs.); Wirth \& Blanton, 1959: 442 (Panama; redescription; distribution; in key; figs.); Franca-Rodríguez, 1963: 67 (Uruguay record); Messersmith, 1964: 339 (Virginia; breeding site); Williams, 1964: 463 (Trinidad; larvae habitat); Smith \& Varnell, 1967: 520 (Florida record; tree holes); Wirth \& Blanton, 1971a: 34 (redescription; distribution; in key; fig.); Wirth \& Blanton, 1971b: 75 (khalafi as synonymy; notes); Wirth \& Blanton, 1973: 431 (Brazil; diagnosis; distribution; in key; fig.); Reeves et al 2004: 9 (USA record; notes).

Culicoides (Oecacta) debilipalpis Lutz: Wirth, 1974: 29 (Catalogue of the Americas south of US); Aitken et al., 1975: 119 (Brazil; debilipalpis group; diagnosis; distribution; fig.); Blanton \& Wirth, 1979: 78 (USA, Florida; diagnosis; distribution; in key; figs.); Vitale et al., 1981: 149 (Panama; in key; diagnosis; fig.).

Culicoides (Haematomyidium) debilipalpis Lutz: Spinelli \& Ronderos, 1997: 304 (revalidation); Borkent \& Spinelli, 2000: 32 (Neotropical catalog); Ronderos et al., 2003: 22 (Yacyretá records); Spinelli \& Borkent, 2004: 391 (Costa Rica records); Spinelli et al., 2005: 141 (Argentina record; in key; fig.); Trindade \& Gorayeb, 2005: 67 (Brazil record); Borkent \& Spinelli, 2007: 66 (Neotropical catalog); Borkent \& Grogan, 2009: 14 (Nearctic catalog); Ronderos et al., 2010: 42 (description immature); Harrup et al., 2015: 252 (fig.); Borkent, 2016: 15 (online the subgeneric classification of species of Culicoides); Spinelli \& Wolff, 2016: 110 (Colombia record); Ronderos et al., 2018: 639 (immature list); Santarém \& Felippe-Bauer, 2020: 22 (Brazilian species); Borkent \& Dominiak, 2020: 112 (World catalogue).

Culicoides ichesi Ronderos \& Spinelli, 1995: 77 (Argentina; Paraguay; Uruguay; female). Ronderos \& Spinelli, 1998: 81 (Yacyretá record; in key).

Culicoides khalafi Beck, 1957: 104 (Florida; male; female; fig.).

Culicoides lahillei: Spinelli \& Wirth, 1986: 62 (synonymy); Wirth et al., 1988: 48 (Wing Atlas of Neotropical species; fig.); Spinelli et al., 1989: 735 (Argentina record); Spinelli \& Ronderos, 1991: 91 (Uruguay record); Spinelli \& Martinez, 1991: 176 (Uruguay; in key); Waller et al., 1990: 352 (French Guiana; redescription; notes); Lamberson et al., 1992: 117 (United States; description; pupa); Borkent \& Wirth, 1997: 72 (synonymy; World species of Ceratopogonidae).

Remarks. Macfie (1948) included the first record of Culicoides debilipalpis from Chiapas, Mexico. Huerta et al. (2012) confirmed Mexico record, based on the revision of specimens from Veracruz and Yucatan deposited in the Florida State Collection of Arthropods, Gainesville, Florida, USA (FSCA).

New record for Oaxaca. Santiago Yaveo (Fig. 4), GPS: 17²0' 8.5” N, 95 41' 38” W; 23 Oct. 2019, col. Benitez-Alva, J.I., Reyes-Garcia, F., trap CDC, 1 female (slide mounted).

Distribution. Widespread from United States (Maryland, Kentucky, Nebraska south to Louisiana and Florida), Mexico (Chiapas, Veracruz, Oaxaca, Yucatan), Guatemala and Belize to Argentina (Macfie, 1948; Borkent \& Spinelli, 2007; Huerta et al., 2012).

\section{Culicoides (Hoffmania) foxi Ortiz}

(Figs. 1A, 4)

Culicoides foxi Ortiz, 1950b: 461 (Puerto Rico). Ortiz, 1951a: 4 (Venezuela record; figs.); Fox, 1953: 888 (Puerto Rico record; notes); Wirth \& Blanton, 1956a: 309 (Panama; redescription; distribution; in key; figs.); Forattini, 1957: 205 (Brazil; diagnosis; distribution; in key; figs.); Fox \& García-Moll, 
1961: 120 (Puerto Rico record; notes); Williams, 1964: 463 (Trinidad; larvae habitat); Aitken et al., 1968: 265 (Trinidad record; habits); Tikasingh, 1972: 447 (Trinidad record; habits); Wirth \& Blanton, 1973: 434 (Brazil; diagnosis; distribution; in key; fig.); Wirth \& Blanton, 1974: 41 (West Indian review; diagnosis; distribution; in key; figs.); Aitken et al., 1975: 124 (Trinidad record; diagnosis; distribution; in key; fig.); Ramírez-Pérez, 1984: 62 (Venezuela record); Greiner et al., 1984: 398 (Bluetongue in the Caribbean region); Spinelli \& Wirth, 1986: 51 (Amazon basin record; in key); Greiner \& Rawlins, 1987: 153 (Jamaica record); Lien \& Lu, 1987: 93 (Bolivia record; fig.); Greiner et al., 1989: 101 (Bluetongue in Trinidad and Tobago); Waller et al., 1990: 357 (French Guiana record; notes); Spinelli \& Wirth, 1993: 34 (Argentina species list; distribution); Borkent \& Wirth, 1997: 68 (World species of Ceratopogonidae); Silva et al., 2001: 353 (Brazil records); Soria et al., 2002: 320 (Brazil, Peru record); Ronderos et al., 2003: 22 (Paraguay record); Laender et al., 2004: 145 (Brazil record); Spinelli et al., 2005: 139 (Argentina record; in key; fig.).

Culicoides (Hoffmania) foxi Ortiz: Wirth \& Blanton, 1959: 283 (Panama; redescription; distribution; in key; figs.); Wirth, 1974: 24 (Catalogue of the Americas south of US); Wirth et al., 1988: 16 (Wing Atlas of Neotropical species; fig.); Spinelli \& Ronderos, 1991: 88 (Argentina, Uruguay record; in key; fig.); Spinelli et al., 1993: 41 (review Neotropical of subgenera Hoffmania; diagnosis; distribution; in key; figs.); Borkent \& Spinelli, 2000: 34 (Catalog of New World, South USA); Borkent \& Spinelli, 2007: 68 (Neotropical catalog); Felippe-Bauer et al., 2008: 35 (Peru record); Huerta et al., 2012: 11 (Mexico records); Borkent, 2016: 17 (online the subgeneric classification of species of Culicoides); Spinelli \& Wolff, 2016: 111 (Colombia); Santarém \& Felippe-Bauer, 2020: 24 (Brazilian species); Borkent \& Dominiak, 2020: 114 (World catalogue).

Culicoides diabolicus Hoffman, of authors, misidentification; Floch \& Abonnenc, 1942: 2 (French Guiana; notes; figs.).

Culicoides guttatus (Coquillett), of authors, misidentification; Fox, 1948: 23 (Venezuela record); Fox, 1949: 31 (Puerto Rico record); Fox \& Kohler, 1950: 342 (Puerto Rico record; biology).

Remarks. This species is common and widely distributed within the Neotropical region. It was previously known in Mexico (Wirth \& Blanton, 1974; Aitken et al., 1975), and subsequently, Spinelli et al., (1993) reported distribution records for this Veracruz, Oaxaca, and Guerrero. Huerta et al. (1912) included new local records from Veracruz and Oaxaca.

New record for Oaxaca. Santiago Yaveo (Fig. 4), GPS: 17²0’ 8.5” N, 95 41’38” W; 20 Nov. 2019, col. Benitez-Alva, J. I., Reyes-Garcia, F., trap CDC, 14 females (slide mounted) (CAIM); Putla Villa de Guerrero, Rancho Viejo, Constancia del Rosario, GPS: 17 04’ 05.8” N, $97^{\circ}$ 59’ 48” W; 16 Dec. 2019 , col. Benitez-Alva, J. I., Zurita-Perez, G., trap CDC, 1 female (slide mounted).

Distribution. Mexico (Guerrero, Chiapas, Oaxaca, Veracruz) through Central and South America to Bolivia and northeastern Argentina; Caribbean area (Jamaica, Trinidad \& Tobago, Puerto Rico) (Spinelli et al., 1993; Borkent \& Spinelli, 2007; Huerta et al., 2012).

\section{Culicoides (Hoffmania) hylas Macfie \\ (Figs. 1B, 4)}

Culicoides hylas Macfie, 1940: 26 (British Guiana; female; fig.). Macfie, 1948: 70 (Caribbean record; in key); Wirth \& Blanton, 1956b: 98 (Panama; diagnosis; distribution; in key; figs.); Forattini, 1957: 243 (Brazil; diagnosis; distribution; in key; figs.); Borkent \& Wirth, 1997: 70 (World species of Ceratopogonidae).

Culicoides (Hoffmania) hylas: Wirth \& Blanton, 1959: 276 (Panama; diagnosis; distribution; in key; figs.); Wirth \& Blanton, 1968: 203 (Neotropical review hylas group; diagnosis; distribution; in key; figs.); Wirth \& Blanton, 1973: 439 (Brazil record; diagnosis; distribution; in key; fig.); Wirth, 1974: 25 (Catalogue of the Americas south of US); Wirth et al., 1988: 18 (Wing Atlas of Neotropical species; 
fig.); Borkent \& Spinelli, 2000: 34 (Catalog of New World, South USA); Borkent \& Spinelli, 2007: 68 (Neotropical catalog); Felippe-Bauer et al., 2008: 260 (Peru record; in key); Felippe-Bauer et al., 2009: 855 (Brazil; diagnosis; distribution; in key); Huerta et al., 2012: 11 (Mexico record); Borkent, 2016: 17 (online the subgeneric classification of species of Culicoides); Spinelli \& Wolff, 2016: 112 (Colombia record); Ronderos et al., 2018: 640 (immature list); Santarém \& Felippe-Bauer, 2020: 25 (Brazilian species); Borkent \& Dominiak, 2020: 114 (World catalogue).

Remarks. This is a common species in the Neotropical region (Borkent \& Spinelli, 2007). It is known in Mexico, south from the state Veracruz (Wirth \& Blanton, 1968; Huerta et al., 2012).

New record for Oaxaca. Santiago Yaveo (Fig. 4), GPS: 17²0' 8.5” N, 95 41'38” W; 20 Nov. 2019, col. Benitez-Alva, J. I., Reyes-Garcia, F., trap CDC, 23 females ( 5 females slide mounted, rest preserved in ethyl alcohol), 5 males (slide mounted); same date except, 23 Oct. 2019, 1 male (slide mounted).

Distribution. Mexico south (Oaxaca, Veracruz) to Colombia, Guyana, Peru, and Brazil (Wirth \& Blanton, 1968; Borkent \& Spinelli, 2007; Huerta et al., 2012).

\section{Culicoides (Hoffmania) insignis Lutz}

\section{(Fig. 1D, 4)}

Culicoides insignis Lutz, 1913: 51 (Brazil; male; female; pupae; figs.). Costa-Lima, 1937: 415 (notes on Lutz collection; fig.); Floch \& Abonnenc, 1942: 1 (French Guiana record; fig.); Vargas, 1945: 43 (Mexico record); Barbosa, 1947: 20 (Brazil; in key; fig.); Fox, 1948: 25 (diagnosis); Macfie, 1948: 75 (Mexico record; in key); Vargas, 1949: 200 (list species); Barbosa, 1952: 17 (Brazil record); Forattini et al. 1956: 195 (Brazil; immature stages; figs.); Forattini, 1957: 223 (Brazil; diagnosis; distribution; in key; figs.); Williams, 1964: 463 (Trinidad; larvae habitat); Linley, 1965: 57 (Jamaica; pupa; fig.); Cavalieri \& Chiossone, 1966: 148 (Argentina record); Davies, 1967: 39 (Jamaica record; habitat); Gutsevich et al., 1969: 4 (Cuba record); Davies \& Giglioli, 1977: 414 (Cayman Island record); Davies \& Giglioli, 1979: 593 (Cayman Island record); Wirth \& Soria, 1981: 107 (Brazil record); Barreto, 1986: 144 (Colombia catalog); Gibbs et al., 1989: 141 (USA, Florida; virus isolated); Waller et al., 1990: 358 (French Guiana record); Homan et al., 1990: 1089 (potential bluetongue vector); Tanya et al., 1992: 1 (bluetongue virus); Borkent \& Wirth 1997: 71 (World species of Ceratopogonidae).

Culicoides (Hoffmania) insignis Lutz: Fox, 1948: 25 (designation type species of Hoffmania); Wirth \& Blanton, 1956a: 319 (Panama; designated male syntype as lectotype; USA, Florida record; synonymy: inamollae, painteri); Wirth \& Blanton, 1959: 285 (Panama; guttatus group; diagnosis; distribution; in key; figs.); Wirth \& Blanton, 1973: 440 (Brazil; diagnosis; distribution; in key; fig.); Wirth, 1974: 25 (Catalogue of the Americas south of US; synonymy); Wirth \& Blanton, 1974: 57 (West Indian review; diagnosis; distribution; in key; figs.); Aitken et al., 1975: 130 (Trinidad record; distribution; in key; fig.); Blanton \& Wirth, 1979: 106 (USA, Florida; diagnosis; distribution; in key; figs.); Ramírez-Pérez, 1984: 62 (Venezuela record); Greiner et al., 1984: 389 (Bluetongue in the Caribbean region); Wirth et al., 1985: 12 (Wing Atlas of Nearctic species; fig.); Wilkening et al., 1985: 520 (USA, Florida records); Spinelli \& Wirth, 1986: 52 (in key; Amazon basin record; fig.); Greiner \& Rawlins, 1987: 153 (Jamaica record); Lien \& Lu, 1987: 94 (Bolivia record; fig.); Wirth et al., 1988: 16 (Wing Atlas of Neotropical species; fig.); Greiner et al., 1989: 101 (Bluetongue in Trinidad and Tobago); Greiner et al., 1990: 289 (Bluetongue in Caribbean region); Spinelli \& Ronderos, 1991: 85 (Argentina, Uruguay records; in key; fig.); Spinelli \& Wirth, 1993: 34 (Argentina list; distribution); Spinelli et al., 1993: 53 (review Neotropical of subgenera Hoffmania; diagnosis; distribution; in key; figs.); Spinelli, 1998: 325 (Argentina record); Ronderos \& Spinelli, 1998: 79 (Paraguay record; in key); Borkent \& Spinelli, 2000: 34 (Catalog of New World, South USA); Spinelli \& Borkent, 2004: 391 (Costa Rica record); Spinelli \& Ronderos, 2005: 63 (Medical 
importance; fig.); Spinelli et al., 2005: 139 (Argentina record; in key; fig.); Borkent \& Spinelli, 2007: 68 (Neotropical catalog); Borkent \& Grogan, 2009: 14 (Nearctic catalog); Spinelli et al., 2009: 88 (Colombia record); Ronderos et al., 2011: 1188 (Argentina list); Huerta et al., 2012: 12 (Mexico record); Marino et al. 2013: 784 (pupae description); Borkent, 2016: 17 (online the subgeneric classification of species of Culicoides); Vigil et al., 2014: 7 (United States record; distribution); Spinelli \& Wolff, 2016: 112 (Colombia record); Santarém \& Felippe-Bauer, 2020: 25 (Brazilian species); Borkent \& Dominiak, 2020: 114 (World catalogue).

Culicoides guttatus (Coquillett): misidentification Lutz, 1913: 58 (Brazil; fig.); Beck, 1952: 102 (Florida records).

Culicoides inamollae Fox \& Hoffman, 1944: 110 (Puerto Rico record; figs.). Barbosa, 1947: 9 (in key); Fox, 1948: 25 (United States, Florida record; fig.); Fox \& Kohler, 1950: 341 (Puerto Rico record; biology); Fox, 1953: 888 (Puerto Rico record; notes); Foote \& Pratt, 1954: 25 (United States record; diagnosis; distribution; in key; fig.); Fox, 1955: 242 (synonymy: painteri, oliveri [sic]); Beck, 1958: 9 (Florida record); Fox \& García-Moll, 1961: 120 (Puerto Rico record; notes).

Culicoides oliveri Fox \& Hoffman, 1944: 108 (in part, diagnosis; Haiti record; figs.).

Culicoides painteri Fox, 1946: 257 (Honduras record; fig.). Fox, 1948: 26 (Florida record; fig.); Barbosa, 1947: 9 (in key); Ortiz, 1950a: 451 (notes).

Remarks. This species is known as possible vector of bluetongue virus in the Neotropical Region (Sáenz \& Greiner, 1994; Borkent, 2005). This species is known as widely distributed in the Neotropical region, and also throughout southeastern USA (Borkent \& Grogan, 2009). It was previously known in Mexico from Chiapas and Yucatan (Macfie, 1948; Blanton \& Wirth, 1979; Borkent \& Spinelli, 2007). Huerta et al. (2012) provided records from Tabasco and Veracruz.

New record for Oaxaca. Santiago Yaveo (Fig. 4), GPS: 17²0' 8.5” N, 95 41' 38” W; 20 Nov. 2019, col. Benitez-Alva, J. I., Reyes-Garcia, F., trap CDC, 1 female (slide mounted).

Distribution. United States (Alabama, Georgia, Florida), Mexico (Chiapas, Oaxaca, Veracruz, Tabasco, Yucatan), Central American and Caribbean to central Argentina (Macfie, 1948; Blanton \& Wirth, 1979; Borkent \& Spinelli, 2007; Huerta et al., 2012).

\section{Culicoides (Hoffmania) ocumarensis \\ (Figs. 2A, 4)}

Culicoides ocumarensis Ortiz, 1950a: 455 (Venezuela; male, female; figs.). Ortiz \& León, 1954: 571 (Ecuador record; notes; fig.); Lien \& Lu, 1987: 93 (Bolivia record; fig.). Borkent \& Wirth, 1997: 77 (World species of Ceratopogonidae).

Culicoides (Hoffmania) ocumarensis Ortiz: Wirth, 1974: 25 (Catalogue of the Americas south of US); Wirth et al., 1988: 18 (Wing Atlas of Neotropical species; fig.); Spinelli et al., 1993: 63 (review Neotropical of subgenera Hoffmania; diagnosis; distribution; in key; figs.); Borkent \& Spinelli, 2000: 34 (Catalog of New World, South USA); Borkent \& Spinelli, 2007: 69 (Neotropical catalog); Spinelli et al., 2009: 88 (Colombia record); Borkent, 2016: 17 (online the subgeneric classification of species of Culicoides); Spinelli \& Wolff, 2016: 112 (Colombia record); Santarém \& Felippe-Bauer, 2020: 25 (Brazilian species); Borkent \& Dominiak, 2020: 114 (World catalogue).

Culicoides diabolicus Hoffman, of authors, misidentification; Macfie, 1932: 488 (Colombia record; notes); Wirth \& Blanton, 1956a: 316 (synonymy: ocumarensis); Wirth \& Blanton, 1959: 280 (synonymy).

Culicoides lutzi Costa-Lima, of authors, in part; Forattini, 1957: 238 (synonymy; diagnosis; distribution; in key; figs.).

Culicoides insignis Lutz, of authors, misidentification; Floch \& Abonnenc, 1942: 2 (male; French Guiana record; figs.); Barbosa, 1944: 259 (Brazil; female; male; figs.).

Culicoides recifei Barbosa, 1947: 25 (Brazil; female); Barbosa, 1952: 20 (notes valid species). 
Culicoides filariferus Hoffman, of authors, in part: Aitken et al., 1975: 121 (synonymy: ocumarensis; Trinidad record; distribution; fig.).

Remarks. This species is widely distributed in the Neotropical region. Previously records confirmed in Mexico included Oaxaca (Istmo de Tehuantepec) and Tabasco (Spinelli et al., 1993).

New record for Oaxaca. Santiago Yaveo (Fig. 4), GPS: 17²0' 8.5” N, 95 41'38” W; 20 Nov. 2019, col. Benitez-Alva, J. I., Reyes-Garcia, F., trap CDC, 1 female, 1 male (slide mounted).

Distribution. Mexico (Oaxaca, Tabasco) to Colombia, Venezuela, Peru, and northern Brazil (Spinelli et al., 1993; Borkent \& Spinelli, 2007).

\section{Culicoides (Hoffmania) pseudodiabolicus Fox}

(Figs. 1C, 4)

Culicoides pseudodiabolicus Fox, 1946: 256 (Trinidad; female; figs.); Barbosa, 1947: 8 (in key); Macfie, 1948: 70 (in key); Aitken et al., 1975: 137 (Trinidad record; distribution; fig.); Wirth \& Blanton, 1973: 445 (Brazil; distribution); Borkent \& Wirth, 1997: 79 (World species of Ceratopogonidae).

Culicoides (Hoffmania) pseudodiabolicus Fox: Wirth \& Blanton, 1959: 283 (Panama; diagnosis; distribution; key; figs.); Wirth, 1974: 24 (Catalogue of the Americas south of US); Wirth et al., 1988: 18 (Wing Atlas of Neotropical species; fig.); Spinelli et al., 1993: 71 (review Neotropical of subgenera Hoffmania; diagnosis; distribution; key; figs.); Borkent \& Spinelli, 2000: 35 (Catalog of New World, South USA); Borkent \& Spinelli, 2007: 69 (Neotropical catalog); Borkent, 2016: 17 (online the subgeneric classification of species of Culicoides); Spinelli \& Wolff, 2016: 113 (Colombia record); Santarém \& Felippe-Bauer, 2020: 25 (Brazilian species); Borkent \& Dominiak, 2020: 115 (World catalogue).

Culicoides diabolicus Hoffman; of authors, misidentification; Macfie, 1937: 7 (in part, Trinidad record; notes); Macfie, 1938: 164 (Trinidad record); Adamson, 1939: 81 (Trinidad; habitats); Macfie, 1940: 25 (British Guiana; as guttatus; synonymy: diabolicus); Adamson, 1941: 74 (Trinidad record; habitats); Wirth, 1956: 249 (Brazil record; pollination); Wirth \& Blanton, 1956b: 316 (in part female; diagnosis; description; in key; figs.); Wirth \& Blanton, 1959: 280 (in part, Panama; diagnosis; distribution; in key; fig.); Williams, 1964: 463 (Trinidad; larvae habitat); Aitken et al., 1968: 265 (Trinidad; habitats); Wirth et al., 1968: 132 (Panama record); Greiner et al., 1989: 103 (Bluetongue in Trinidad and Tobago).

Culicoides trinidadensis Hoffman; of authors, misidentification; Myers, 1935: 71 (in part; Trinidad record). Culicoides guttatus (Coquillett); of authors, misidentification; Macfie, 1938: 166 (in part; Trinidad record).

Remarks. The species is included in a species group complex -Culicoides filariferus, C. pseudodiabolicus, C. diabolicus and C. guttatus- by Aitken et al. (1975) and is the most difficult taxonomic group in the genus (Spinelli et al., 1993). Previously records from Mexico need to be confirmed because most of the records are indicated as $C$. diabolicus and $C$. filariferus, in which several species were usually misidentified (Spinelli et al., 1993). At the moment, the confirmed record in Mexico from for locality of Puente Nacional (Veracruz). The specimens of the subgenus Hoffmania of Colección de Artrópodos con Importancia Médica (CAIM) are in review.

New record for Oaxaca. Oaxaca, Santiago Yaveo (Fig. 4), GPS: $17^{\circ} 20^{\prime} 8.5^{\prime \prime}$ N, 95 $41^{\circ} 38^{\prime \prime} \mathrm{W}$; 20 Nov. 2019, col. Benitez-Alva, J. I., Reyes-Garcia, F., trap CDC, 4 females (slide mounted), same date except, 23 Oct. 2019, 6 females, 10 males (slide mounted).

Distribution. Mexico (Veracruz, Oaxaca) to Peru and north and northeastern of Brazil (Borkent \& Spinelli, 2007). 
ACKNOWLEDGMENTS. We thank to Dra. Maria Luiza Felippe-Bauer, FIOCRUZ, Brazil, for support of the identification of Culicoides ginesi and for their helpful review of an earlier draft of the manuscript. Also, to Programa de Vectores of the state of Oaxaca and Dr. Fabian Correa-Morales CENAPRECE for their support in the collection.

\section{LITERATURE CITED}

Adamson, A. M. (1939) Observations of biting sandflies (Ceratopogonidae) in Trinidad, British West Indies. Tropical Agriculture Trinidad, 16, 79-81.

Adamson, A. M. (1941) Observations of biting sandflies (Ceratopogonidae) in Trinidad, British West Indies. Caribbean Medical Journal, 3, 69-76.

Aitken, T. H. G., Worth, C.B., Tikasingh, E. S. (1968) Arbovirus studies in Bush Forest, Trinidad. W. I., September 1959-December 1964. III. Entomological studies. American Journal Tropical Medicine Hygiene, 17, 253-268. https://doi.org/10.4269/ajtmh.1968.17.253

Aitken, T. H. G., Wirth, W. W., William, R. W., Davis, J. B., Tikasingh, E. S. (1975) A review of the bloodsucking midges of Trinidad and Tobago, West Indies (Diptera: Ceratopogonidae). Journal of Entomology B, 44 (2), 101-144. https://doi.org/10.1111/j.1365-3113.1975.tb00007.x

Atchley, W. R. (1967) The Culicoides of New Mexico (Diptera: Ceratopogonidae). Kansas University Science Bulletin, 46, 937-1022.

Barbosa, F. A. S. (1944) "Culicoides insignis" Lutz, com a descricao do hipopigio (Diptera, Chironomidae). Revista Brasilileira do Biologia, 4, 259-261.

Barbosa, F. A. S. (1947) Culicoides (Diptera: Heleidae) da regiao neotropica. Annais da Sociedade de Biologia de Pernambuco, 7, 3-30.

Barbosa, F. A. S. (1952) Novos subsidies para o conhecimento dos Culicoides Neotropicos (Diptera: Heleidae) Impresa Industrial Recife, $21 \mathrm{pp}$.

Barreto, P. (1986) Catálogo de los Culicoides (Diptera: Ceratopogonidae) de Colombia. Colombia Medico, $17,140-150$.

Battle, F. V., Turner, E. C. (1970) Culicoides (Diptera: Ceratopogonidae) reared from breeding site collections in North Carolina with a summary of the species occurring in that state. Mosquito News, $30,425-427$.

Battle, F. V., Turner, E. C. (1971) The Insects of Virginia No. 3. A systematic review of the genus Culicoides (Diptera: Ceratopogonidae) of Virginia with a geographic catalog of the species occurring in the eastern United State north of Florida. Virginia Polytechnic Institute and State University, Research Division Bulletin 44, 1-129.

Beck, E. C. (1952) Notes on the distribution of Culicoides in Florida (Diptera: Ceratopogonidae). Florida Entomologist, 35, 101-107.

Beck, E. C. (1956) A new species of Culicoides from Florida with additional distribution data for the genus (Diptera: Heleidae). Florida Entomologist, 39, 133-138. https://doi.org/10.2307/3492428

Beck, E. C. (1957) Two new species of Culicoides from Florida (Diptera: Heleidae). Florida Entomologist, 40, 103-105. https://doi.org/10.2307/3492509

Beck, E. C. (1958) A population study of the Culicoides of Florida (Diptera: Heleidae). Mosquito News, $18,6-11$.

Biagi, F., Tay, J., De Biagi, de B. A. M. (1958) Observaciones sobre mansonelosis en la Peninsula de Yucatán, Culicoides furens como vector. Medicina (Mex)., 38, 377-379.

Blanton, F. S., Wirth, W. W. (1979) The sand flies (Culicoides) of Florida (Diptera: Ceratopogonidae). Arthropods of Florida and Neighboring Land Areas, 10, 1-204. 
Borkent, A. (2005) The Biting Midges, Ceratopogonidae (Diptera). Chapter 10. Pp: 113-126. In: W. C. Marquardt (Ed.), Biology of Disease Vectors. 2nd Edition. Elsevier Academic Press, Burlington, Massachusetts, $\mathrm{i}-\mathrm{xxiii}+1-785 \mathrm{pp}$.

Borkent, A. (2014) The pupae of the biting midges of the world (Diptera: Ceratopogonidae), with a generic key and analysis of the phylogenetic relationships between genera. Zootaxa, 3879, 1-327.

Borkent, A. (2016) The subgeneric classification of species of Culicoides. Available: http://www.inhs.uiuc.edu/ research/FLYTREE/CulicoidesSubgenera.pdf (last access 15 November 2019).

Borkent, A. (2017) Ceratopogonidae (biting midges). Chapter 34. Pp: 733-812. In: A. H. Kirk-Spriggs, B. J. Sinclair (Eds.). Manual of Afrotropical Diptera. Volume 2. Nematocerous Diptera and lower Brachycera. Suricata 5. South African National Biodiversity Institute, Pretoria.

Borkent, A., Dominiak, P. (2020) Catalog of the biting midges of the world (Diptera: Ceratopogonidae). Zootaxa, 4787 (1), 001-377.

Borkent, A., Grogan, Jr. W. L. (2009) Catalog of the New World biting midges north of Mexico (Diptera: Ceratopogonidae). Zootaxa, 2273, 1-48. https://doi.org/10.11646/zootaxa.2273.1.1

Borkent, A., Spinelli, G. R. (2000) Catalog of the new world biting midges south of the United States of America (Diptera: Ceratopogonidae). Contributions on Entomology International, 4, 1-107.

Borkent, A., Spinelli, G. R. (2007) Neotropical Ceratopogonidae (Diptera: Insecta). Pp:1-198. In: J. Adis, J. R. Arias, G. Rueda-Delgado, K. M. Wantzen (Eds.). Aquatic biodiversity in Latin America (ABLA). Vol. 4. Pensoft, Sofia-Moscow.

Borkent, A., Spinelli, G. R., Grogan, Jr. W. L. (2009) Ceratopogonidae (biting midges, purrujas). Chapter 29. Pp: 407-435. In: B. V. Brown, A. Borkent, J. M. Cumming, D. M. Wood, N. E. Woodley, M. A. Zumbado (Eds.). Manual of Central American Diptera. Volume 1. NRC Research Press, Ottawa, Ontario, Canada.

Borkent, A., Wirth, W. W. (1997) World species of biting midges (Diptera: Ceratopogonidae). Bulletin of the American Museum of Natural History, 233, 1-257.

Cavalieri, F., Chiossone, I. F. (1966) Sobre el conocimiento actual del género hematófago Culicoides Latreille, 1809, en la Argentina (Diptera, Ceratopogonidae). Physis, 86, 145-153.

Cochrane, A. H. (1974) Two new Nearctic species of Culicoides (Diptera: Ceratopogonidae). Florida Entomologist, 56, 311-318.

Costa-Lima, A. Da. (1937) Chave das especies de Culicoides de regioa neotropica (Diptera: Ceratopogonidae). Memorias do Institute Oswaldo Cruz, 32, 411-432.

Dampf, A. (1936) Los ceratopogónidos o jejenes (Insecta, Diptera, Fam. Ceratopogonidae) como transmisores de filarias. Medicina, 268, 1-7.

Davies, J. B. (1967) The distribution of sandflies (Culicoides spp.) breeding in a tidal mangrove swamp in Jamaica and the effect of tides on the emergence of $C$. furens Poey and C. barbosai Wirth \& Blanton. West Indian Medical Journal, 41, 39-50.

Davies, J. E., Giglioli, M. E. C. (1977) The breeding sites and seasonal occurrence of Culicoides furens in Grand Cayman with notes on the breeding sites of C. insignis (Diptera: Ceratopogonidae). Mosquito News, 37, 414-423.

Davies, J. E., Giglioli, M. E. C. (1979) The Ceratopogonidae (Diptera) of Grand Cayman, West Indies: species and ecological notes. Mosquito News, 39 (3), 586-594.

Edwards, F. W. (1922) On some Malayan and other species of Culicoides, with a note on the genus Lasiohelea. Bulletin Entomological Research, 15, 161-167.

Felippe-Bauer, M. L., Cáceres, A. G., Santos da Silva, C., Valderrama-Bazan, W., Gonzales-Pérez, A., Costa, J. M. (2008) Description of Culicoides pseudoheliconiae sp. n. from Peruvian Amazon and revalidation of Culicoides contubernalis Ortiz \& León (Diptera: Ceratopogonidae). Memorias do Instituto Oswaldo Cruz, 103, 259-262.

https://doi.org/10.1590/S0074-02762008005000010 
Felippe-Bauer, M. L., Cáceres, A. G., Santos da Silva, C., Valderrama-Bazan, W., Gonzales-Pérez, A., Martins, C. J. (2008) New records of Culicoides Latreille (Diptera: Ceratopogonidae) from Peruvian Amazonian region. Biota Neotropica, 8 (2), 34-38.

Felippe-Bauer, M. L., Pinto-Damasceno, C., Py-Daniel, V., Spinelli, G. R. (2009) Culicoides baniwa sp. nov. from the Brazilian Amazon Region with a synopsis of the hylas species group (Diptera: Ceratopogonidae). Memorias do Instituto Oswaldo Cruz, 104 (6), 851-857. https://doi.org/10.1590/S0074-02762009000600005

Floch, H., Abonnec, E. (1942) Ceratopogonides hematophages de la Guyane Francaise. Publication Institut Pasteur de la Guyane et du Territorre de L'Inini, 57,1-10.

Foote, R. H., Pratt, H. D. (1954) The Culicoides of the eastern United States. Public Health Monographic, $18,1-55$.

Forattini, O. P. (1957) Culicoides da região neotropical (Diptera, Ceratopogonidae). Arquivos da Faculdade de Higiene e Saúde Pública da Universidade de São Paulo, 11, 159-526. https://doi.org/10.11606/issn.2358-792X.v11i2p161-526

Forattini, O. P, Rabello, E. X., Pattoli, D. (1956) Nota sobre a larva e pupa de Culicoides insignis Lutz, 1913 (Diptera, Ceratopogonidae). Revista Brasileira de Entomologia, 4, 195-198.

Fox, I. (1946) A review of the species of biting midges or Culicoides from the Caribbean Region (Diptera: Ceratopogonidae). Annals of the Entomological Society of America, 39, 248-258. https://doi.org/10.1093/aesa/39.2.248

Fox, I. (1948) Hoffmania, a new subgenus in Culicoides (Diptera: Ceratopogonidae). Proceedings of the Biological Society of Washington, 61, 21-28.

Fox, I. (1949) Notes on Puerto Rican biting midges or Culicoides (Diptera: Ceratopogonidae). Bulletin Brooklyn Entomology Society, 44, 29-34.

Fox, I. (1953) Light trap studies on Culicoides in Puerto Rico. Journal Economic Entomology, 45, 888889.

Fox, I. (1955) A catalogue of the bloodsucking midges of the Americas (Culicoides, Leptoconops, and Lasiohelea) with keys to the subgenera and Nearctic species, a geographic index, and bibliography. Journal of the University of Puerto Rico, 39, 214-285.

Fox, I., García-Moll, I. (1961) The Culicoides of the International Airport, Isla Verde, Puerto Rico, as shown by light traps. Mosquito News, 21, 120-132.

Fox, I., Hoffman, W. A. (1944) New neotropical biting sandflies of the genus Culicoides (Diptera: Ceratopogonidae). Puerto Rico Journal Public Health \& Tropical Medicine, 80, 108-111.

Fox, I., Kohler, C. E. (1950) Distribution and relative abundance of the species of biting midges or Culicoides in eastern Puerto Rico, as shown by light traps. Puerto Rico Journal Public Health Tropical Medicine, 25, 342-349.

Franca-Rodríguez, M. E. (1963) Primeros estudios sobre los Culicoides (Diptera: Ceratopogonidae. Revista Uruguaya de Patología Clínica, 1, 65-70.

Gazeau, L. J., Messersmith, D. H. (1970) Rearing and distribution of Maryland Culicoides (Diptera: Ceratopogonidae). Mosquito News, 30, 30-34.

Gibbs, E. P. J., Calisher, C. H., Tesh, R. B., Lazuick, J. S., Bowen, R., Greiner, E. C. (1989) Bivens arm virus: A new rhabdovirus isolated from Culicoides insignis in Florida and related to Tibrogargan virus of Australia. Veterinary Microbiology, 19, 141-150.

Greiner, E. C., Alexander, F. C. M., Roach, J., Moe, V., Borde, G., Taylor, W. P., Dickinson, J., Gibbs, E. P. J. (1989) Bluetongue epidemiology in the Caribbean region: serological and entomological findings from a pilot sentinel system in Trinidad and Tobago. Medical and Veterinary Entomology, 3, 101-105.

Greiner, E. C., Alexander, F. C. M., Roach, J., St. John, V. S., King, T. H., Taylor, W. P., Gibbs, E. P. J. (1990) Bluetongue epidemiology in the Caribbean region: serological and entomological evidence from a pilot study in Barbados. Medical and Veterinary Entomology, 4, 289-295. 
Greiner, E. C., Garris, G. I., Rollo, R. T., Knausenberger, W. L., Jones, J. E., Gibbs, E. P. J. (1984) Preliminary studies on the Culicoides spp. as potential vectors of bluetongue in the Caribbean region. Preventive Veterinary Medicine, 2, 398-399.

Greiner, E. C., Rawlins, C. (1987) Culicoides spp. collected near ruminants in Jamaica and their relevance to bluetongue. Journal of Agriculture Entomology, 4, 153-156.

Gutsevich, A. V., Garcia-Avila, I., Gonzalez-Broche, R. (1969) Resultado del estudio sobre los jejenes hematófagos (Diptera, Ceratopogonidae) en Cuba. Torreia, 16, 3-7.

Harrup, L. E, Bellis, G. A., Balenghien, T., Garros, C. (2015) Culicoides Latreille (Diptera: Ceratopogonidae) taxonomy: Current challenges and future directions. Infection, Genetics and Evolution, 30, 249-266. https://doi.org/10.1016/j.meegid.2014.12.018

Hoffman, W. A. (1925) A review of the species of Culicoides of North and Central America and the West Indies. American Journal of Hygiene, 5, 274-301. Available: http://europepmc.org/search?query=AUTH:\%22Homan\%20EJ\%22

Homan, C. L., Thompson, L. H., Barreto, C. H., Oviedo, M. T., Gibbs, E. P. J., Greiner, E. C. (1990) Epidemiologic study of bluetongue viruses in Central America and the Caribbean: 1986-1988. Regional Bluetongue Team. American Journal of Veterinary Research, 51 (7), 1089-1094.

Huerta, H., Rodríguez-Castrejón, A. M., Grogan, Jr. W. L., Ibáñez-Bernal, S. (2012) New records of biting midges of the genus Culicoides Latreille from Mexico (Diptera: Ceratopogonidae). Insecta Mundi, 0211, 1-20.

Ibáñez-Bernal, S., Wirth, W. W., Huerta, H. (1996) Ceratopogonidae (Diptera), Capítulo 36. Pp: 567577. In: J. Llorente-Bousquets, A. García-Aldrete, E. González-Soriano, E. (Eds.). Biodiversidad, taxonomía y biogeografía de artrópodos de México: "Hacia una sintesis de su conocimiento". Universidad Nacional Autónoma de México, Ciudad de México.

Jamnback, H. (1965) The Culicoides of New York state (Diptera: Ceratopogonidae). New York State Museum and Science Service Bulletin, No 399, viii + 154 pp.

Kettle, D. S. (1977) Biology and bionomics of bloodsucking ceratopogonids. Annual Review Entomology, $22,33-51$.

Khalaf, K. T. (1969) Distribution and phenology of Culicoides (Diptera: Ceratopogonidae) along the Gulf of Mexico. Annals Entomology of Society American, 62, 1153-1161.

Laender, J. O., Ribeiro, E. S., Guimaraes, A. M., Portela, Z. I., Felippe-Bauer, M. L. (2004) Levantamento das especies de Culicoides Latreille,1809 (Diptera: Ceratopogonidae) encontradas nas mesorregioes norte de Minas Jequitinhonha e vale do mucura, Minas Gerais, Brasil. Entomologia \& Vectores, 11, 145-157.

Lamberson, C., Pappas, C. D., Pappas, L. G. (1992) Pupal taxonomy of the tree-hole Culicoides (Diptera: Ceratopogonidae) in Eastern North America. Annals of the Entomological Society of America, 85 (2), 111-120.

Lien, J. I. H., Lu, Liang-Chen. (1987) A small collection of biting midges of the genus Culicoides from Bolivia (Diptera, Ceratopogonidae). Journal Taiwan Museum, 40, 91-100.

Linley, J. R. (1965) Description of the pupae of Culicoides barbosai, C. insignis, and C. borinqueni (Diptera: Ceratopogonidae). Annals of the Entomological Society of America, 58, 57-63.

Linley, J. R., Hoch, A. L., Pinheiro, F. P. (1983) Biting midges (Diptera: Ceratopogonidae) and human health. Journal of Medical Entomology, 20 (4), 347-364.

Lutz, A. (1913) Contribuição para o estudo das Ceratopogoninas hematofagas do Brazil. Parte sistematica. Segunda Memoria. Memorias do Instituto Oswaldo Cruz, 5, 45-73.

Macfie, J. W. S. (1932) Some new or Little-known Ceratopogonidae. Annals Magazine Natural History, serie 10 (9), 485-499. https://doi.org/10.1080/00222933208673523

Macfie, J. W. S. (1937) Ceratopogonidae from Trinidad. Annals. Museum Natural History, 10, 1-18. https://doi.org/10.1080/00222933708655311 
Macfie, J. W. S. (1938) Notes on Ceratopogonidae (Diptera). Proceedings of the Royal Entomological Society of London (B), 7, 157-166. https://doi.org/10.1111/j.1365-3113.1938.tb01271.x

Macfie, J. W. S. (1940) A report on a collection of Ceratopogonidae (Diptera) from British Guiana. Entomologist's Monthly Magazine, 76, 23-32.

Macfie, J. W. S. (1948) Some species of Culicoides (Diptera, Ceratopogonidae) from the state of Chiapas, Mexico. Annals of Tropical Medicine and Parasitology, 42, 67-87. https://doi.org/10.1080/00034983.1948.11685349

Marino, P. I., Cazorla, G. C., Ronderos, M. M. (2011) Study of the immature stages of two species of the biting midges genus Culicoides (Diptera: Ceratopogonidae). Acta Entomologica Musei Nationalis Pragae, 53, 777-792.

Mellor, P. S., Boorman, J., Baylis, M. (2000) Culicoides biting midges: Their role as arbovirus vectors. Annual Review of Entomology, 45, 307-340.

Messersmith, D. H. (1964) The breeding site of Culicoides debilipalpis Lutz (Diptera: Ceratopogonidae) in Virginia. Mosquito News, 24, 339.

Messersmith, D. H. (1966) Report on a collection of Culicoides (Diptera: Ceratopogonidae) from western Virginia. Virginia Journal Science, 17, 83-104.

Mukhopadhyay, E., Mazumdar, A., Joardar, S. N., Goutam, S. K., Banerjee, D. (2016) An annotated checklist of Culicoides Latreille, 1809 (Insecta: Ceratopogonidae: Diptera) with incorporation of a vector species list from India. Journal of Vector Ecology, 41 (2), 279-284.

Myers, J. G. (1935) The sand-fly pest (Culicoides). Tropical Agriculture Trinidad, 12, 71-73.

Ortiz, I. (1950a) Estudios en Culicoides IV. Revisión de las especies americanas del subgénero Hoffmania Fox 1948, con la descripción de dos nuevas especies. Revista de Sanidad y Asistencia Social, 15, 437-460.

Ortiz, I. (1950b) Estudios en Culicoides V. Informes sobre una nueva especie y lista de los machos cuyas genitalias son conocidas. Revista de Sanidad y Asistencia Social, 15, 461-465.

Ortiz, I. (1951a) Estudio sobre Culicoides (Diptera: Ceratopogonidae) VII. Culicoides dominicii y clave para el reconocimiento de las especies venezolanas. Contribuciones Ocasionales del Museo de Historia Natural, La Salle, Zoología serie, 5, 12 pp.

Ortiz, I. (1951b) Estudios en Culicoides (Diptera: Ceratopogonidae) IX. Sobre los caracteres diferenciales entre Culicoides paraensis (Goeldi, 1905), C. stellifer (Coquillett, 1901), y C. lanei (Ortíz, 1950). Descripción de cuatro nuevas especies con la redescripción de algunas otras poco conocidas. Revista de Sanidad y Asistencia Social, 16, 573-591.

Ortiz, I., León, L. A. (1954) Los Culicoides (Diptera: Ceratopogonidae) de la República del Ecuador. Boletín Informativo Científico Nacional, 67, 564-590.

Ortiz, I., León, L. A. (1955) Los Culicoides (Diptera: Ceratopogonidae) de la República del Ecuador. Boletin de Informaciones Cientificas Nacionales, 67, 564-594.

Ortiz, I., Mirsa, M. (1951) Estudios en Culicoides. Descripción de dos nuevas especies: Culicoides avilaensis y $C$. discrepans, y del macho de $C$. leopoldoi Ortiz, 1951. Redescripción de $C$. limai Barretto, 1944, C. baueri Hoffman 1925, C. lichyi Floch and Abonnenc, 1949, y C. pusillus Lutz, 1913. Revista de Sanidad y Asistencia Social, 16, 593-605.

Ortiz, I., Mirsa, M. (1952) Culicoides de Venezuela. - Redescripción de 10 especies con la descripción de algunos sexos no conocidos. Revista de Sanidad y Asistencia Social, 17, 257-279.

Ramírez-Pérez, J. (1984) Revisión de los dípteros hematófagos del género Culicoides en Venezuela. Boletín de la Dirección de Malariología de Sanidad Ambiental, 24, 49-70.

Reeves, W. K., Adler, P. H., Grogan, Jr. W. L., Super, P. E. (2004) Hematophagous and parasitic Diptera (Insecta) in the Great Smoky Mountains National Park, USA. Zootaxa, 483, 1-44.

Ronderos, M. M., Spinelli, G. R. (1995) Redescripción de Culicoides lahillei y descripción de Culicoides ichesi, n. sp. de la Argentina, Paraguay y Uruguay (Diptera: Ceratopogonidae). Neotrópica, 41, 7781. 
Ronderos, M. M., Spinelli, G. R. (1998) Las especies de Culicoides en el área de influencia de la represa de Yacyretá (Diptera: Ceratopogonidae). Revista de la Sociedad Entomológica de Argentina, 57 (14), 79-83.

Ronderos, M. M., Greco, N. M., Spinelli, G. R. (2003) Diversity of biting midges of the genus Culicoides Latreille (Diptera: Ceratopogonidae) in the Area of the Yacyretá Dam Lake between Argentina and Paraguay. Memorias do Instituto Oswaldo Cruz, 98 (1), 19-24.

Ronderos, M. M., Cazorla, C. G., Spinelli, G. R. (2010) The immature stages of the biting midge Culicoides debilipalpis Lutz (Diptera: Ceratopogonidae). Zootaxa, 2716, 42-52.

Ronderos, M. M., Díaz, F., Marino, P. I., Ferreira-Keppler, R. L. (2018) Family Ceratopogonidae, Chapter 16.1. Pp: 625-659. In: H. Neusa, H. James, J. H. Thorp, D. C. Rogers (Eds.). Keys to Neotropical Hexapoda Thorp and Covich's Freshwater Invertebrates - Volume III. Fourth Edition, Academic Press Elsevier, USA.

Ronderos, M. M., Marino, P. I., Florentina, D., Estevez, L. A. (2011) Biting midge (Diptera: Ceratopogonidae) from Matín García Island, Argentina. Revista de Biologia Tropical, 59, 1183 1194.

Root, D. S., Gerhardt, R. R. (1991) Seasonal emergence patterns of Culicoides (Diptera: Ceratopogonidae) in eastern Tennessee. Journal of Agriculture Entomology, 8, 127-135.

Root, F. M., Hoffman, W. A. (1937) The North American species of Culicoides. American Journal of Hygiene, 25, 150-176.

Sáenz, M. R., Greiner, E. C. (1994) Culicoides aspirated from cattle in Costa Rica, Honduras, Panama and Puerto Rico and their role as potential vectors of bluetongue viruses. Medical and Veterinary Entomology, 8, 15-19.

Santarém, M. C. A., Felippe-Bauer, M. L. (2020) Brazilian species of biting midges -espécies de maruins do Brasil (Diptera: Ceratopogonidae). Available: https://portal.fiocruz.br/sites/portal.fiocruz.br/files/documentos/brazilian_species_of_biting_midg es_2019.pdf (last access 24 June 2020).

Silva, C. S., Felippe-Bauer, M. L., Almeida, E. H. G., Figueiredo, L. R. (2001) Culicoides (Diptera: Ceratopogonidae) do estado do Rio de Janeiro, Brasil. I. Região Norte: Município de Campos do Goytacazes. Entomologia \& Vectores, 8 (3), 349-358.

Smith, W. W., Varnell, J. H. Jr. (1967) Hydrogen ion concentration (pH) as related to the occurrence and abundance of tree-holes dwelling Culicoides spp. (Diptera: Ceratopogonidae) in northern Florida. Mosquito News, 27, 519-521.

Soria, S. J., Felippe-Bauer, M. L., Oliveira, S. J. (2002) Lista das espécies de Ceratopogonidae (Diptera: Ceratopogonidae) do agroecosistema cacaueiro, depositadas na Coleção Entomológica do Instituto Oswaldo Cruz, Rio de Janeiro, Brasil. Entomologia \& Vectores, 9 (3), 317-327.

Spinelli, G. R. (1998) Ceratopogonidae. Pp: 314-326. In: J. J. Morrone, S. Coscaron (Eds.). Biodiversidad de Artrópodos Argentinos. Una perspectiva biotaxonómica. Ediciones Sur, La Plata Argentina.

Spinelli, G. R., Borkent, A. (2004) New species of Central American Culicoides Latreille (Diptera: Ceratopogonidae) with a synopsis of species from Costa Rica. Proceedings of the Entomological Society of Washington, 106, 361-395.

Spinelli, G. R., Greiner, E. C., Wirth, W. W. (1993) The Neotropical bloodsucking midges of the Culicoides guttatus group of the subgenus Hoffmania (Diptera: Ceratopogonidae). Contributions of the American Entomological Institute, 27 (3), 1-91.

Spinelli, G. R., Huerta, H. (2015) Four new species of Mesoamerican biting midges of the genus Culicoides (Diptera: Ceratopogonidae). Acta Entomologica Musei Nationalis Pragae, 55 (2), $811-$ 824.

Spinelli, G. R., Martinez, M. (1991) The genus Culicoides in Uruguay (Diptera: Ceratopogonidae). Insecta Mundi, 5, 175-179.

Spinelli, G. R., Ronderos, M. M. (1991) Los polvorines del género Culicoides en áreas de influencia de la represa de Salto Grande (Diptera: Ceratopogonidae). Neotrópica, 37, 83-94. 
Spinelli, G. R., Ronderos, M. M. (1997) Description of the male and revalidation of Culicoides debilipalpis (Diptera: Ceratopogonidae). Transactions of the American Entomological Society, 123 (4), 303309.

Spinelli, G. R., Ronderos, M. M. (2005) Ceratopogonidae (formas hematófagas). Pp: 61-66. In: O. D. Salomon (Ed.). Artrópodos de interés medico en Argentina, Buenos Aires, Argentina, 120 pp.

Spinelli, G. R., Ronderos, M. M., Balseiro, E. G. (1989) Análisis de la actividad diaria de dípteros nematóceros en Punta Lara (Pdo. Ensenada, Prov. de Buenos Aires). II. Ceratopogonidae, con la descripción de una especie nueva del género Atrichopogon. Limnobios, 2, 733-737.

Spinelli, G. R., Ronderos, M. M., Díaz, F., Marino, P. I. (2005) The bloodsucking biting midges of Argentina (Diptera: Ceratopogonidae). Memorias do Instituto Oswaldo Cruz, 100, 137-150.

Spinelli, G. R., Santamaría, E., Cabrera, O. L., Ronderos, M. M., Suárez, M. F. (2009) Five new species of Culicoides Latreille described from Colombia, yielding a new species list and country records (Diptera: Ceratopogonidae). Memorias do Instituto Oswaldo Cruz, 104, 81-92.

Spinelli, G. R., Wirth, W. W. (1986) Clave para la identificación de las especies del género Culicoides Latreille presentes al sur de la cuenca amazónica. Nuevas citas y notas sinonímicas (Diptera: Ceratopogonidae). Revista de la Sociedad Entomológica Argentina, 44, 49-73.

Spinelli, G. R., Wirth, W. W. (1993) Los Ceratopogonidae de la Argentina (Insecta: Diptera). Pp: 124. In: Z. Castellanos (Ed.). Fauna de agua dulce de la República Argentina. Profadu-Conicet, Volumen 38, Argentina.

Spinelli, G. R., Wolff, M. (2016) Family Ceratopogonidae.Pp: 98-141. In: M. Wolff, S. S. Nihei, C. J. B. De Carvalho (Eds.). Catalogue of Diptera of Colombia. Zootaxa, 4122 (1), 1-949. https://doi.org/10.11646/zootaxa.4122.1.15

Tanya, V. N., Greiner, E. C., Gibbs, E. P. J. (1992) Evaluation of Culicoides insignis (Diptera: Ceratopogonidae) as a vector of bluetongue virus. Veterinary Microbiology, 32 (1), 1-14.

Tikasingh, E. S. (1972) Seasonal and diurnal activities of four species of Trinidadian Culicoides (Diptera: Ceratopogonidae). Mosquito News, 32 (3), 447-452.

Trindade, R. L., Gorayeb, I. S. (2005) Maruims (Ceratopogonidae: Diptera) do Estuario do Río Pará e do Litoral do Estado do Pará, Brasil. Entomología y Vectores, 12 (1), 61-74.

Vargas, L. (1945) Nota sobre Ceratopogonidos y Culicoides. Revista del Instituto de Salubridad y Enfermedades Tropicales, 4, 41-49.

Vargas, L. (1949) Lista de los Culicoides del mundo (Diptera: Heleidae). Revista de la Sociedad Mexicana de Historia Natural, 10, 191-218.

Vargas, L. (1953) Culicoides wirthomyia n. sp. y Culicoides stigmalis Wirth 1952. Revista del Instituto de Salubridad y Enfermedades Tropicales, 13, 227-233.

Vargas, L. (1954) Dos nuevas especies de Culicoides Mexicanos. Revista del Instituto de Salubridad y Enfermedades Tropicales, 14, 25-32.

Vargas, L. (1960) The subgenera of Culicoides of the Americas (Diptera, Ceratopogonidae). Revista de Biologia Tropical, 8, 35-47.

Vigil, S. L., Wlodkowski, J. C., Parris, J., de Vargas, E. S., Shaw, D., Cleveland, C., Grogan, Jr. W. L., Corn, J. L. (2014) New records of biting midges of the genus Culicoides Latreille from the southeastern United States (Diptera: Ceratopogonidae). Insecta Mundi, 0394, 1-14.

Vitale, G. C., Wirth, W. W., Aitken, T. H. G. (1981) New species and records of Culicoides reared from arboreal habitats in Panama, with a synopsis of the debilipalpis group (Diptera: Ceratopogonidae). Proceedings of the Entomological Society of Washington, 83, 140-159.

Waller, J., Lancelot, R., Lefevre, P. C., Kremer, M. (1990) Note faunistique sur les Culicoides de Guyane (Diptera: Ceratopogonidae). Revue d'élevage et de Medicine Vétérinaire des pays tropicaux, 43, 349-364.

Williams, R. W. (1955) Studies on the Culicoides of Baker Country, Georgia (Diptera, Heleidae). I. Preliminary survey and observations. Annals of Entomology Society of America, 48, 30-34.

Williams, R. W. (1964) Observations on habitats of Culicoides larvae in Trinidad, W. I. (Diptera: Ceratopogonidae). Annals of Entomology Society of American, 57, 462-466. 
Wilkening, A. J., Kline, D. L., Wirth, W. W. (1985) An Annotated checklist of the Ceratopogonidae (Diptera) of Florida with a new synonymy. Florida Entomologist, 68 (4), 511-537.

Winder, J. A. (1977) Field observations on Ceratopogonidae and other Diptera: Nematocera associated with cocoa flowers in Brazil. Bulletin Entomology Research, 67, 57-63.

Winder, J. A., Silva, P. (1972) Cacao pollination: Microdiptera of cacao plantations and some of their breeding places. Bulletin Entomology Research, 61, 651-655.

Wirth, W. W. (1955a) Three new species of Culicoides from Texas (Diptera, Heleidae). Journal of the Washington Academy of Sciences, 45, 355-359.

Wirth, W. W. (1955b) Report on a collection of biting midges of the genus Culicoides from Guatemala (Diptera, Heleidae). Proceedings of the Entomological Society of Washington, 57, 109-117.

Wirth, W. W. (1956) The heleid midges involved in the pollination of rubber trees in America. Proceedings Entomology Society of Washington, 55, 241-250.

Wirth, W. W. (1965) Family Ceratopogonidae. Pp: 121-142. In: A. Stone, C. W. Sabrosky, W. W. Wirth, R. H. Foote, J. R. Coulson (Eds.). A catalog of the Diptera of America north of Mexico. United States Department of Agriculture, Agricultural Research Service, Agriculture Handbook 276: i-iv + 1-1696 pp.

Wirth, W. W. (1974) Family Ceratopogonidae. In: Papavero, N. (Ed.). A catalogue of the Diptera of the Americas south of the United States. Fasc., 14, 1-89.

Wirth, W. W., Blanton, F. S. (1953) Studies in Panama Culicoides (Diptera, Heleidae) III. A new species related to phlebotomus (Williston). Entomological News, 64, 113-120.

Wirth, W. W., Blanton, F. S. (1956a) Studies in Panama Culicoides VIII. The Neotropical species of the guttatus group of the subgenus Hoffmania (Diptera, Heleidae). Proceedings of the Entomological Society of Washington, 58, 305-326.

Wirth, W. W., Blanton, F. S. (1956b) Studies in Panama Culicoides (Diptera: Heleidae) VI. The hylas group of the subgenus Hoffmania. Journal of Washington Academic Science, 5, 95-99.

Wirth, W. W., Blanton, F. S. (1956c) Studies in Panama Culicoides (Diptera, Heleidae) IX. Two new species related to leoni Barbosa and reevesi Wirth. Bulletin of the Brooklyn Entomological Society, $51,45-52$.

Wirth, W. W., Blanton, F. S. (1959) Biting midges of the genus Culicoides from Panama (Diptera: Heleidae). Proceedings of the United States National Museum, 109, 237-482. https://doi.org/10.5479/si.00963801.109-3415.237

Wirth, W. W., Blanton, F. S. (1968) A revision of the Neotropical biting midges of the hylas group of Culicoides (Diptera, Ceratopogonidae). Florida Entomologist, 51, 201-215. https://doi.org/10.2307/3493420

Wirth, W. W., Blanton, F. S. (1969) North America [sic] Culicoides of the pulicaris group (Diptera: Ceratopogonidae). The Florida Entomologist, 52, 207-243. https://doi.org/10.2307/3493875

Wirth, W. W., Blanton, F. S. (1971a) New Neotropical sandflies of the Culicoides debilipalpis group (Diptera: Ceratopogonidae). Proceedings of the Entomological Society of Washington, 73, 34-43.

Wirth, W. W., Blanton, F. S. (1971b) New species and synonymy of Florida Culicoides (Diptera: Ceratopogonidae). Florida Entomologist, 54, 73-78.

https://doi.org/10.2307/3493791

Wirth, W. W., Blanton, F. S. (1973) A review of the maruins or biting midges of the genus Culicoides (Diptera: Ceratopogonidae) in the Amazon Basin. Amazoniana, 4, 405-470.

Wirth, W. W., Blanton, F. S. (1974) The West Indian sandflies of the genus Culicoides (Diptera: Ceratopogonidae). Agricultural Research Service U.S.D.A. Technical Bulletin 1474: iv + 98 pp.

Wirth, W. W., Dyce, A. L., Peterson, B. V. (1985) An atlas of wing photographs, with a summary of the numerical characters of the Nearctic species of Culicoides (Diptera: Ceratopogonidae). Contributions of the American Entomological Institute, 22, 1-46. 
Wirth, W. W., Dyce, A. L., Spinelli, G. R. (1988) An atlas of wing photographs, with a summary of the numerical characters of the Neotropical species of Culicoides (Diptera: Ceratopogonidae). Contributions of the American Entomological Institute, 25, 1-72.

Wirth, W. W., Hubert, A. A. (1960) Ceratopogonidae (Diptera) reared from cacti, with a review of the copiosus group of Culicoides. Annals of the Entomological Society of America, 53, 639-658.

Wirth, W. W., Mullens, B. A. (1992) Culicoides boydi (Diptera: Ceratopogonidae): A potential vector of hemorrhagic disease viruses to Desert Bighorn Sheep in Southern California. Journal of Medical Entomology, 29 (6), 1006-1010. https://doi.org/10.1093/jmedent/29.6.1006

Wirth, W. W., Soria, S. G. (1981) Two Culicoides biting midges reared from inflorescences of Calathea in Brazil and Colombia and a key to the species of the discrepans group (Diptera: Ceratopogonidae). Revista Theobroma, 11, 107-117.

Wirth, M. O., Wirth, W. W., Blanton, F. S. (1968) Plant materials as breeding places of Panama Culicoides (Diptera: Ceratopogonidae). Proceedings of the Entomological Society of Washington, $70,132$. 